\title{
Average Waiting Time Profiles of Uniform DQDB Model $\dagger$
}

\author{
Nageswara S.V. Rao $\S$ \\ Intelligent Systems Section \\ Center for Engineering Systems Advanced Research \\ Oak Ridge National Laboratory \\ Oak Ridge, TN 37831-6364
}

Kurt Maly, Steve Olariu, Sudheer Dharanikota, Liping Zhang, David Game Department of Computer Science

Old Dominion University

Norfolk, VA 23529-0162

September 7, 1993

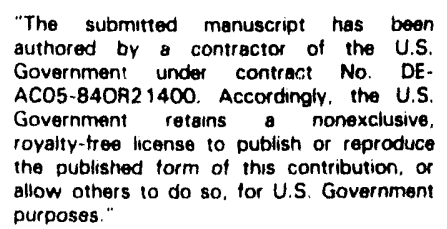

Submitted to: IEEE INFOCOM'94: Thirteenth Annual Joint Conference of the IEEE Computer and Communications Societies.

$\S$ Research of the first author is sponsored by the Engineering Research Program of the Office of Basic Energy Sciences, of the U.S. Department of Energy, under Contract No. DE-AC05-840R21400 with Martin Marietta Energy Systems, Inc. Part of the work was performed while the author was with the Department of Computer Science, Old Dominion University, Norfolk, VA 23j29-0162.

tThis project is partially funded by Sun Microsystems Inc. under the grant \#596044, Taurus Technologies Inc. under the grant \#596046, NASA under the grant \#187263 and Virginia's Center for Innovative Technology under the grant \#596045. 


\section{Contents}

$\begin{array}{lr}\text { Abstract } & 3\end{array}$

1 Introduction 4

2 DQDB Model $\quad 7$

3 Working-Window Concept 8

3.1 Oncoming Jobs . . . . . . . . . . . . . . . . . . . 9

3.2 Accumulated Request Bits . . . . . . . . . . . . . . . . 10

4 Average Waiting time 10

4.1 Profiles of $\delta_{R}(i) \ldots \ldots \ldots \ldots \ldots \ldots$

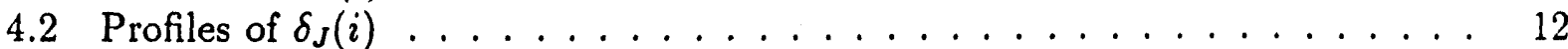

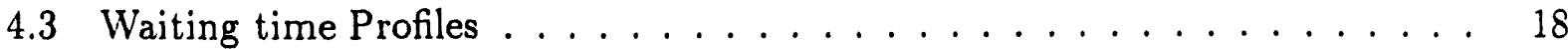

4.3.1 Expected Waiting time . . . . . . . . . . . . . . 19

4.3 .2 Low to Medium Loads . . . . . . . . . . . . . . . . . . 20

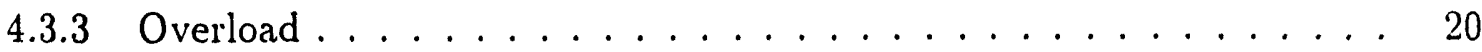

4.3 .4 Medium to High Loads . . . . . . . . . . . . . . . . . . 20

4.3 .5 High to Overload . . . . . . . . . . . . . . . 22

5 Simulation Model $\quad 22$

6 Semblance of Chaos $\quad 24$

7 General DQDB Model 26

8 Conclusions $\quad 27$

$\begin{array}{ll}\text { Appendix A: Profiles of } \delta_{R}(i) & 27\end{array}$

$\begin{array}{ll}\text { Appendix B: Profiles of } \xi(i, x) & 28\end{array}$

$\begin{array}{lr}\text { Appendix C } & 29\end{array}$

$\begin{array}{ll}\text { Appendix D: Two Systems of Equations } & 30\end{array}$

Appendix E: Coarse Analysis for $W_{J}(i) \quad 30$

$\begin{array}{ll}\text { References } & 33\end{array}$

\section{DISCLAIMER}

This report was prepared as an account of work spensored by an agency of the United States Government. Neither the United States Government nor any agency thereof, nor any of their employees, makes any warranty, express or implied, or assumes any legal liability or responsibility for the accuracy, cornpleteness, or usefulness of any information, apparatus, product, or process disclosed, or represents that its use would not iniringe privately owned rights. Reference herein to any specific commercial product, process, or service by trade name, trademark, manufacturer, or otherwise does not necessarily constitute or imply its endorsement, recommendation, or favoring by the United States Government or any agency thereof. The views and opinions of authors expressed herein do not necessarily state or reflect those of the United States Government or any agency thereof. 


\begin{abstract}
The Distributed Queue Dual Bus (DQDB) system consists of a linear arrangement of $N$ nodes that communicate with each other using two contra-flowing buses; the nodes use an extremely simple protocol to send messages on these buses. This simple, but elegant, system has been found to be very challenging to analyze. We consider a simple and uniform abstraction of this model to highlight the fairness issues in terms of average waiting time. We introduce a new approximation method to analyze the performance of DQDB system in terms of the average waiting time of a node expressed as a function of its position. Our approach abstracts the intimate relationship between the load of the system and its fairness characteristics, and explains all basic behavior profiles of DQDB observed in previous simulation. For the uniform DQDB with equal distance between adjacent nodes, we show that the system operates under three basic behavior profiles and a finite number of their combinations that depend on the load of the network. Consequently, the system is not fair at any load in terms of the average waiting times. In the vicinity of a critical load of $1-4 / N$, the uniform network runs into a state akin to chaos, where its behavior fluctuates from one extreme to the other with a load variation of $2 / N$. Our analysis is supported by simulation results. We also show that the main theme of the analysis carries over to the general (non-uniform) DQDB; by suitably choosing the inter-node distances, the DQDB can be made fair around some loads, but such system will become unfair as the load changes.
\end{abstract}

Keywords and Phrases: network protocols, DQDB, working window, distributed queue, performance analysis 


\section{Introduction}

High speed Metropolitan Area Networks (MAN) are presently being developed to accommodate high transmission speeds over large geographic areas. Two major MAN architectures that have been recently studied are: (a) ANSI X3T9.5 FDDI ring [1] and (b) IEEE 802.6 dualbus [2, 25]. The latter, called Distributed Queue Dual Bus (DQDB), is the subject of study in this paper; early accounts of this system (originally called QPSX) are described in $[29,30]$. An account of the evolution of DQDB is presented in [33]. Our focus is to analyze the fairness issues of a simplified version of this system when viewed as a special type of distributed system controlled by a very simple protocol. Our main thesis is to establish, using analytical methods, that "DQDB-like" systems are unlikely to be fair, in terms of average waiting times, over large load variations. In particular, we consider a simple and uniform approximation to the DQDB where the propagation time between adjacent nodes is a unit slot time. We show that even such a simple system exhibits a variety of waiting time distributions (described as a function of the location of the nodes) as the load is changed; our analysis shows that this uniform DQDB is not fair at any load. The general DQDB system, however, can possibly be made fair around some loads by using a particular assignment of distances between adjacent nodes, but such system is shown to become unfair over large load changes. Our motivation is to illustrate the unfairness behavior of DQDB-like systems rather than to accurately capture the behavior of an actual DQDB system; in this vein, our focus is one of analysis rather than precise modeling.

There have been several efforts to analyze the performance of DQDB both by using analytical models and simulation studies (see [27] for a comprehensive survey of DQDB literature). A complete analysis that takes into account detailed aspects of DQDB seems to be analytically intractable mainly due to the intricate interaction of a number of dynamic variables. Consequently, various researchers concentrate on specific aspects of DQDB by suitably abstracting a set of appropriate properties. There seems to be a general consensus that this system has rather severe problems of fairness $[8,15,14,19,20,21,34]$; many of these conclusions are based on simulation results. In this paper, we provide analytical evidence to support this claim.

Analytical approaches have modeled DQDB to various degrees of precision. In [34], the throughput of DQDB is derived for a network of two users. In [36,37], the throughput and waiting time characteristics of DQDB have been investigated in the presence of both packet and circuit switched traffic. The performance of DQDB under overload traffic conditions is presented in [38, 39]. In [5], the study has been concentrated on a specific node in the system, and the packet waiting time characteristics of that node have been computed. A single-server queueing system with two classes of customers and state-dependent priorities is proposed in [6]; this model is shown to be useful in modeling a single node of a DQDB system. An embedded model composed of basic $M / G / 1$ modules and station-dependent random process is proposed for approximate analysis of DQDB protocol in [18]. A discrete-time Markov chain model is used in [26] to obtain fairly accurate estimates for throughput and mean segment delay under known loading patterns; this method, however, is computationally intensive for systems with a large number of nodes. A study of effects of overloads on DQDB is performed by $[38,39]$. Simulation studies of this protocol can be found in $[9,10,28,34]$. Various methods have been proposed to overcome the fairness problems $[13,19,16,21,26]$ and to 


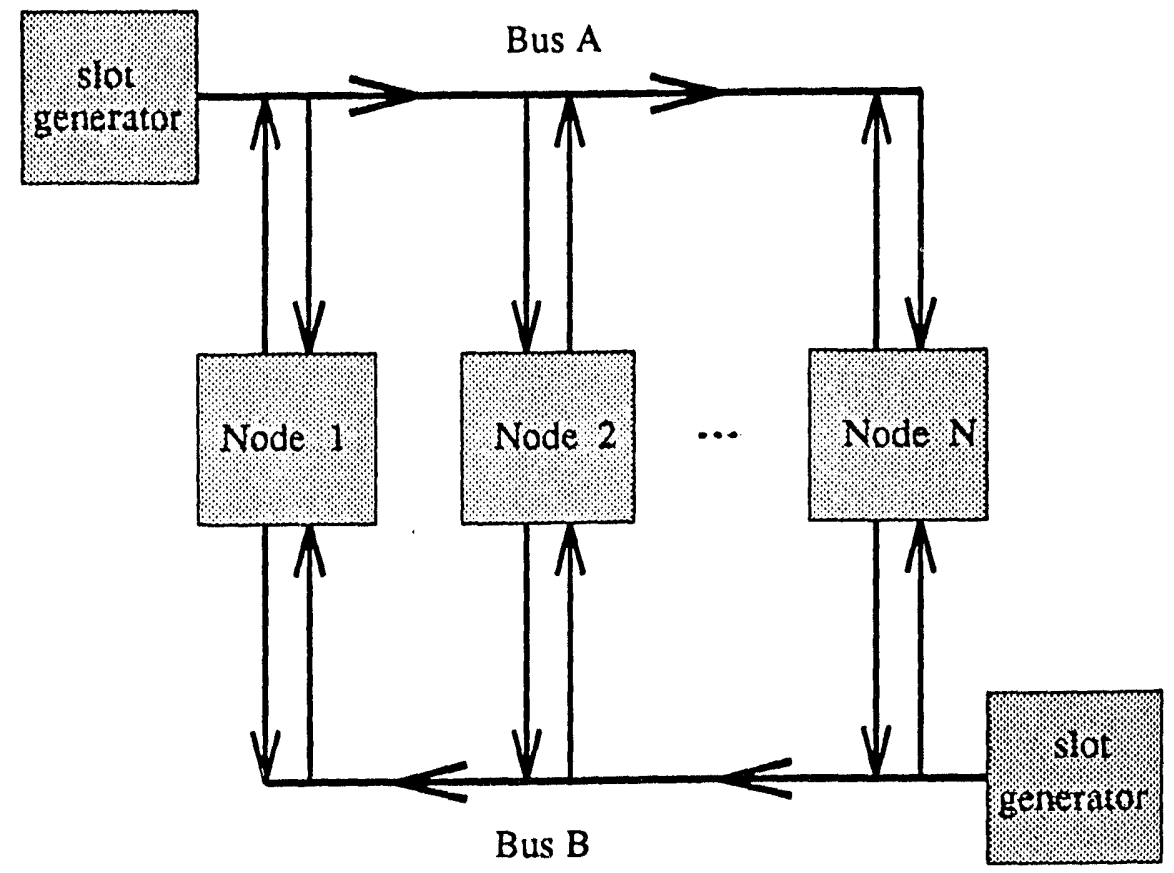

Figure 1: Bus Structure of DQDB.

provide performance improvements by employing some modifications [26, 24, 23, 32, 35].

In this paper, we concentrate on the global issues of fairness of a basic version of DQDB in terms of average waiting times at the individual nodes. More specifically, we investigate how the profiles of average waiting time change as functions of load; using a single model, we explain several seemingly different profiles observed at different loads during simulation studies.

In DQDB, a number of nodes are arranged in a linear manner with two unidirectional buses carrying slots in two opposite directions (Fig. 1). External messages arrive at the nodes and are destined to other nodes. The messages are put into appropriate slots in the buses by using a rather simple distributed queuing mechanism. In order to send a job down stream on a bus, a node performs the following actions: (i) sends a request upstream using the other bus; (ii) waits until a suitable number of free slots have passed by; and (iii) waits for the next free slot and sends the message (more details are presented in next section). There is an extremely tricky dynamic interaction between the jobs arriving at the various nodes and the jobs flowing in the buses. As a result, this system generates extremely complicated dynamics in terms of the actual waiting times of various jobs.

We assume that the external jobs at any node arrive according to a Poisson distribution, and the jobs are uniformly destined across the nodes. Majority of the paper deals with the uniform $D Q D B$ model where the distance between two adjacent nodes is one slot time (if this distance is a constant multiple of a slot time then the present analysis is valid). We then illustrate that the fairness problems carry over to the general case ${ }^{1}$. We assume that

\footnotetext{
${ }^{1}$ We conceivably have the possibility of adapting the DQDB protocol (e.g. delaying some jobs at certain nodes longer than their specified time) to the changes in the load such that the system is fair at all loads. But such method requires accurate estimation of load and the waiting time behavior at that load, and such
} 


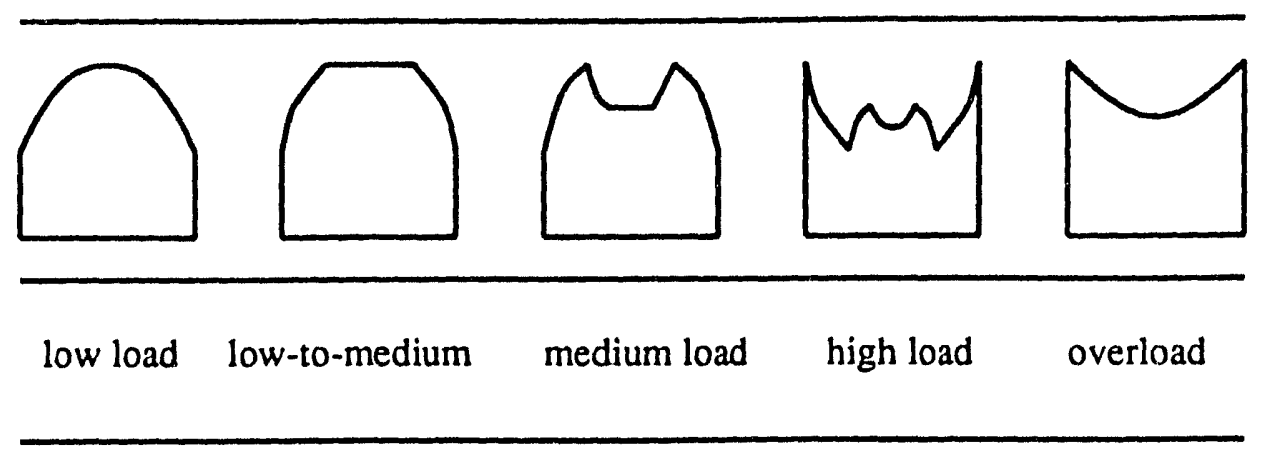

Figure 2: Evolution of waiting time profile with load.

the system has been in operation for some time so that the initial transients have died down and the system is in a probabilistic steady state.

The average waiting time of a job at a node plotted as a function of the node position in the DQDB is referred to as the profile of the system. Under probabilistically uniform job arrivals at nodes, there are two profoundly distinct profiles of the system: at low loads the waiting time is increasing from the end stations to the middle, and at high loads the average waiting time is decreasing from the ends to the middle. This behavior has been reported by several authors based on simulation studies. We propose an approximate analytical model that explains these extremities in the profiles and also the transition from one to the other as the load changes. The evolution of the waiting time profiles with the increase in load for the uniform DQDB model (with jobs being uniformly destined across the nodes of the network) is summarized in Fig. 2 ; here $x$-axis corresponds to the node number and $y$-axis corresponds to the average weighting time. Also in Fig. 2, only the overall shapes of the profile are indicated, and the different profiles are not drawn to the same scale. At low loads, the profile is a dome or convex, and it becomes a bowl or concave at high loads; in the interim, the profile goes through a finite number of shapes.

Our method is based on a notion similar to the working set method proposed to characterize the behavior of programs [11,12]: we use approximations to compute an "average impedance" a job faces at any node, and then derive the profiles by a detailed analysis of various cases of average impedance. Our interest is to illustrate how the profiles vary with the load, and we do not address the seemingly more difficult problem of obtaining accurate estimators for waiting times.

At low loads, the stations located towards the end will have an advantage over the stations in the middle. As we increase the load, this situation reverses to a state where the stations at the end will have longer delays compared to those in the middle. By using a coarser model for the uniform DQDB with $N$ nodes, we show that it only takes a load change of $2 / N$ to cause this inversion when the network is operating around a critical load of $1-4 / N$. In the vicinity of a critical load, slight fluctuations in load make the performance unpredicatable along the lines of chaos in non-linear dynamic systems. Our simulation results show that the system indeed enters such a state around the critical load, where the average waiting time varies rather erratically across the network.

This paper is organized as follows. We define a model which is a uniform and simplified

accuracy is beyond the scope of the analysis of this paper. 
version of the IEEE 802.6 standard DQDB protocol in Section 2. The working set model is presented as an approximate method to analyze DQDB in Section 3. In Section 4, we present the waiting time analysis of the DQDB system. Simulation model is described in Section 5. The unpredictability of the system around the critical load is illustrated in Section 6. Discussion about the applicability of the present analysis to the general "non-uniform" DQDB model is presented in Section 7.

\section{DQDB Model}

The DQDB model ${ }^{2}$ consists of a number of nodes or stations, denoted by $1,2, \ldots, N$, arranged in a linear fashion as shown in Fig. 1. There are two unidirectional buses $A$ and $B$ that support communication in opposite directions. Slots are generated at the start of each bus and then are sent down the bus at a deterministic rate of $N \lambda / 2$ for each bus. External messages arrive at each node according to a Poisson arrival process with a rate of $\lambda$ at the full load. We assume that each message or a job is of the same length as a slot; this is a simplifying assumption to make the analysis tractable. A job arriving at node $i$ is destined to the other nodes according to the uniform probability distribution. For the majority of this paper, we consider the uniform $D Q D B$ model where the electronic distance between every pair of adjacent nodes is one time unit given by $1 /(N \lambda)$. We characterize the average load on the network by a loading fraction or load such that at load $f$, the jobs at each node arrive a rate of $f \lambda$.

If the nodes are freely allowed to acquire any empty slot passing by, stations located closest to the slot generator could acquire all slots and prevent other nodes from transmitting. This becomes a significant problem at high loads. To avoid this, the DQDB protocol uses the request bit on the reverse bus to indicate that a segment has been queued for access; there is one request bit with each slot of either bus. Each node maintains two dedicated counters, request counter and countdown counter, for each bus. Any node wishing to send a message on a specific bus writes into the next free request bit on the reverse bus. Further, it transfers the current value of the request counter into the countdown counter.

The countdown counter indicates the number of requests (for access to this specific bus) which have to be satisfied before the segment at the node is sent; the countdown counter is decremented by one for each empty slot detected. When the countdown counter reaches zero, the segment is written into the next empty slot. Meanwhile, the request counter continues to register the requests on the reverse bus. It should be noted that the operations of writing requests and sending segments are independent. That is, the access for segments is not inhibited if the value of the countdown counter is zero but the request associated with the segment has not yet been written onto the reverse bus.

Note that here we use an approximation that results in a simplification of the analysis. In the IEEE 802.6 standard DQDB protocol, the request bit is not written on the reverse

\footnotetext{
${ }^{2}$ We wish to emphasize that we deal with a model that captures some of the basic features of the DQDB system, and do not attempt to obtain an accurate portrayal of all details of DQDB. More detailed issues of this system are available in several papers $[23,29,30,2,33]$. Also there are several variants of this basic model which will present some performance improvements (e.g., $[15,33,26,35])$, and we restrict our attention to only the basic version of DQDB.
} 
bus until the segment reaches the head of the queue. We assume that the request bit could be written as soon as the segment arrives; in the steady state, the average-case behavior of our approximation approaches that of the standard protocol.

As a distributed system, DQDB is an interesting intermediary between a tightly coupled system and a loosely coupled system. Although this protocol is completely distributed in terms of execution, the overall system is very closely controlled by the buses that make it look like a tightly coupled system.

\section{Working-Window Concept}

The average waiting time of a job for node $i$, denoted by $W_{J}(i)$ is the average of time elapsed since the arrival of a job until its dispatch onto the appropriate bus (the quantity $W_{J}(i)$ is based on both buses). Consider that a job $X$ arrives at node $i$. On the average, $X$ waits for a time period of $W_{J}(i)$ before it gets delivered onto the appropriate bus. We call $W_{J}(i)$ the job-window to indicate the fact that the busy slots that pass by node $i$ during the time period $W_{J}(i)$ (since the arrival of $X$ ) will impede the delivery of $X$.

The average inter-arrival time of a node $i, W_{R}(i)$ is the average inter-arrival time between two consecutive jobs at node $i$. By the assumption of Poission arrival, we have $W_{R}(i)=\frac{1}{j \lambda}$ which is the same for all nodes. On the average, the request bits that arrive only in the window $W_{R}(i)$ will impede delivery of the present job $X$. This is because only the request bits that have been accumulated since the arrival of the last job will impede the current job. The $W_{R}(i)$ is called the request window of node $i$. Note that for a viewer observing at the time of arrival of a job, $W_{J}(i)$ corresponds to the period after the arrival whereas $W_{R}(i)$ corresponds to time period prior to the arrival.

Recall that when the job $X$ arrives at node $i$ : (a) we transfer the contents of request counter to the countdown counter and wait until it is counted down to zero, and then (b) wait until an empty slot appears and then dispatch the message. There are two basic factors that impede the delivery of a job.

(a) Oncoming Jobs: After we start counting down the slots, all jobs that arrive at nodes upstream before our request bit percolates to them, will take up the free slots coming towards node $i$.

(b) Accumulated Request Bits: At the arrival time of a job, all the request bits that have been accumulated since the time the last job arrived at node $i$ will prevent the delivery of $X$.

These two factors will generate very complicated dynamic interactions which are not easily captured, in complete generality, by mathematical formulae. We will "approximate" these quantities by suitably averaging in working windows for node $i$ comprising of $W_{J}(i)$ and $W_{R}(i)$. For the sake of illustration in this section, we consider a message at the node $i$ to be delivered to the node $i+j, j>0$. 


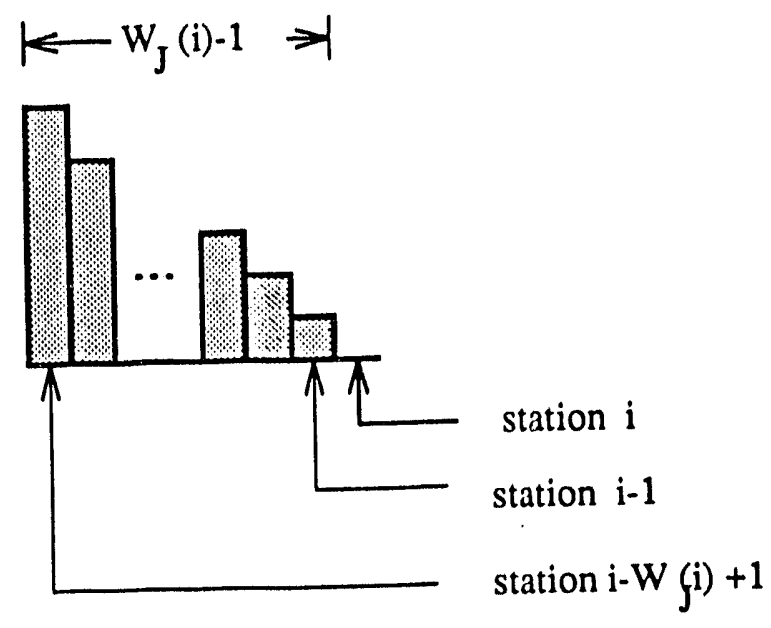

(a) window for jobs

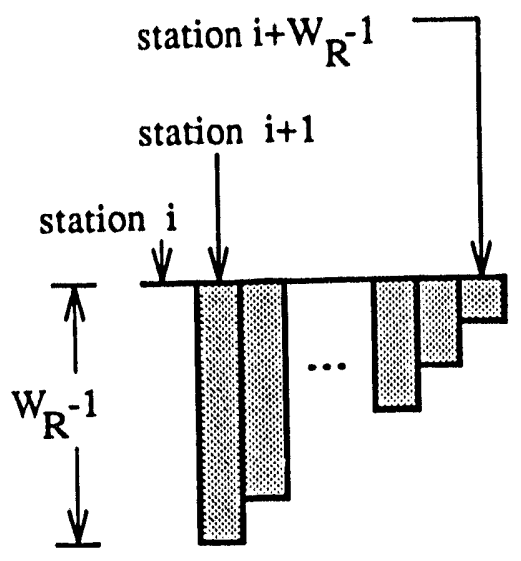

(b) window for request bits

Figure 3: Working windows for request bits and jobs.

\subsection{Oncoming Jobs}

We can imagine a window of size $W_{J}(i)$ for the job $X$ at node $i$ such that the oncoming jobs only during this window will impede $X$ (since $X$ on the average would have been delivered after $W_{J}(i)$ time units by definition). Now examine the jobs that have to be counted in this window by looking at the process by which the request bit corresponding to $X$ gets delivered. Since the slots in the buses have a total rate of $N \lambda$, and the jobs at the nodes arrive at a rate of $f \lambda$, on the average we are more likely to see more slots in the buses (not necessarily empty) than arriving jobs. At each slot, either request bit of the present job gets delivered or that of some other node gets delivered. We can imagine this process as though we deliver the present request bit and keep the bit that has come with the slot. Now imagine that we measure the time in the units of $1 /(f \lambda)$. The request bit of $X$ can be viewed to have been propagated to the station $i-1$ in one unit of time. Moreover, in one time unit and this request bit must have been transferred to the countdown counter since on the average one job must have arrived in unit of time at node $i-1$ which must have flushed the request bit counter at node $i-1$ to the count down counter. Hence, the jobs arriving at station $i-1$ after one unit of time will be impeded by our request bit hence do not count against the delivery of $X$. Similarly, the jobs arriving after two units of time at station $i-2$ will not impede $X$. Hence, the total average impedance to $X$ due to the oncoming jobs is a linear function of the distance of the station position from $i$ in units of $2 /(f \lambda)$. We linearly interpolate this function in between these discrete units at the resolution of the slot time of the buses (given by $2 /(N \lambda)$ ) as shown in Fig. 3(a). This is an approximation because the above argument shows that the number of oncoming jobs to be counted within the window is a linear function only at the steps of $1 /(f \lambda)$, and does not specify its behavior at a finer resolution of step size $1 /(N \lambda)$. 


\subsection{Accumulated Request Bits}

On the average, the request bit counter at each station will be transferred to the count down counter at a probabilistic rate of $f \lambda$ because it is flushed by the arrival of every new job. Thus for job $X$, the window over which the request bits must be counted is $W_{R}(i)=1 /(f \lambda)$ at node $i$. Note that this window size depends on the interarrival times of the jobs which is controlled by the arrival process rather than the dynamics of DQDB. However, the number of request bits that must be counted within this window are decided by the DQDB protocol. We obtain an average value for this quantity as follows. All the jobs that arrive at station $i+1$ within last $W_{R}(i)-1$ units of time, measured in the slot time of the bus, will result in request bits propagated to station $i$, and hence will impede $X$. Similarly the jobs that arrive at station $i+2$ within last $W_{R}(i)-2$ units of time will impede $X$, and so on. Thus the profile of the request bit in this window are shown Fig. 3(a). No ie that this profile is linearly decreasing with respect to distance from $i$ as opposed to the linearly increasing profile of Fig. 3(b).

\section{Average Waiting time}

For a job required to be transmitted at node $i$ we define the impedance factor, $\Delta(i)$, as the sum $^{3}$ of

(a) $\delta_{J}(i)$, the average number of jobs arriving at node $i$ on both buses within the window $W_{J}(i)$, and

(b) $\delta_{R}(i)$, the average number of request bits arriving at node $i$ on both buses within the window $W_{R}(i)$.

Here we do not a priori know which bus the job will be dispatched onto, and the impedence is based on both the buses. Intuitively, $\Delta(i)$ is an average measure of the "impedance" that a job faces when we attempt to put it on the bus, and hence is a measure of probability with which a job gets delivered in a slot. Note that $\Delta(i)$ is only a first order approximation, and a more detailed analysis could unravel more precise weighting schemes ${ }^{4}$. The profiles

\footnotetext{
${ }^{3}$ There are a number of variants of this basis model depending on the degree of approximation. We consider two specific cases. First, the present analysis can be carried out for the impedance of the form $\Delta(i)=a \delta_{R}(i)+b \delta_{J}(i)$ for constants $a, b \geq 0 ;$ in such case, the precise forms of the profiles will depend on $a$ and $b$ but the overall profile evolution from convex to concave will remain the same. For example, in low-to-medium loads the profile will still be convex and in high-to-overloads the profile will still be concave. Second, a more detailed model can be obtained by using $W_{J}^{A}(i)$ and $W_{J}^{B}(i)$ which represent the window sizes corresponding to the buses $A$ and $B$ such that

$$
W_{J}(i)=\operatorname{prob}[A] W_{J}^{A}(i)+\operatorname{prob}[B] W_{J}^{B}(i)
$$

where $\operatorname{prob}[A]$ and $\operatorname{prob}[B]$ denote the probability that the present job is required to be deliveied on bus $A$ and $B$ respectively. Again, the analysis of this paper can be carried out in this case to obtain more detailed profiles, but the overall profiles evolution with load essentially remains the same; a coarse version of similar estimation using prob $[A]$ and $\operatorname{prob}[B]$ is used in Section 6 (derived in Appendix E).

${ }^{4}$ One reason for suspecting unequal weighting scheme is that the request bits seem to have higher precedence since we first wait for request counter to run to zero, before looking for a free slot to dispatch the job. It is also conceivable that the weights are functions of node positions $i$.
} 


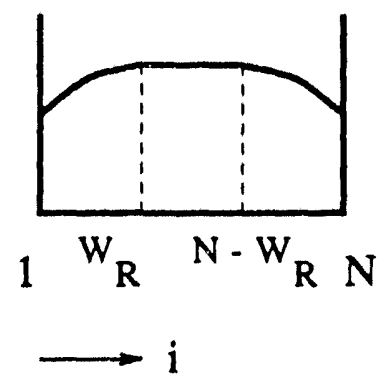

(a) $\mathrm{W}_{\mathrm{R}}<\mathrm{N} / 2$

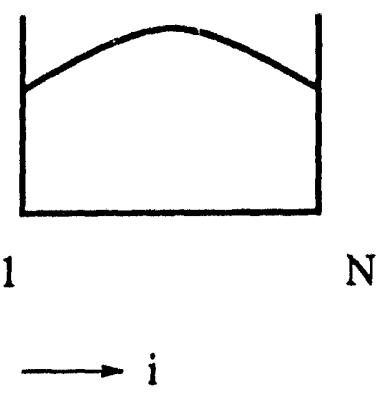

(b) $\mathrm{W}_{\mathrm{R}}>\mathrm{N}$

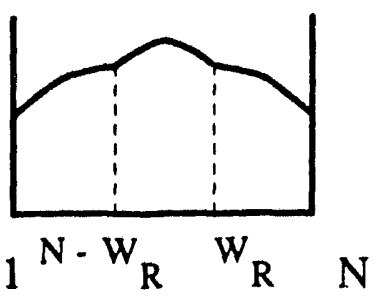

(c) $N / 2 \leq W_{R} \leq N$

Figure 4: Profiles of $\delta_{R}(i)$.

of $\delta_{J}(i)$ and $\delta_{R}(i)$, plotted as functions of $i$ are decided by the load $f$ of the network. We subsequently show that the profile of $\delta_{R}(i)$ is always a dome or convex. But the profile of $\delta_{J}(i)$ transits from a dome (convex) to a bowl (concave) as the load is changed irom low to high.

\subsection{Profiles of $\delta_{R}(i)$}

We have three different cases based on the value of $W_{R}(i)=\frac{1}{\rho \lambda}=W_{R}$ as shown in Fig. 4. Since $\delta_{R}(i)$ is symmetric about the middle node, we present the function in the interval $[1, N / 2]$ for $i$ whenever convenient; in such cases, the profile in the interval $[N / 2, N]$ is a mirror image of the former with respect to the mid point $N / 2$. We only present the results here, and see Appendix A for the proofs.

(a) $W_{R}(i) \leq N / 2$ : This function is monotonically increasing in the range $\left[1, W_{R}\right]$, constant in the range $\left[W_{R}, N-W_{R}\right]$ and monotonically decreasing in the range $\left[N-W_{R}, N\right]$ (Fig. 4(a)). The expression for $\delta_{R}(i)$ is as follows.

$$
\delta_{R}(i)= \begin{cases}W_{R}-1 & \text { if } i \in\left[W_{R}, N / 2\right] \\ W_{R}-1-\frac{L \lambda}{2}\left(W_{R}-i\right)\left(W_{R}-i+1\right) & \text { if } i \in\left[1, W_{R}\right]\end{cases}
$$

(b) $W_{R}(i) \geq N$ : This function is monotonically increasing in the range $[1,(N-1) / 2]$ and is monotonically decreasing in the range $[(N+2) / 2, N]$ (Fig. 4(b)). The expression for $\delta_{R}(i)$ is as follows.

$$
\delta_{R}(i)=N-1-\frac{f \lambda}{\varepsilon}\left[N(N+1)-2(N+1) i+2 i^{2}\right]
$$

(c) $N / 2<W_{R}(i)<N$ : This function is same as that of (a) in the range $\left[1, N-W_{R}\right]$ and as that of (b) in the range $\left[N-W_{R}, N / 2\right]$. Thus it is monotonically increasing until the middle and then monotonically decreasing until the end (Fig. 4(c)). The expression for $\delta_{R}(i)$ is as follows.

$$
\delta_{R}(i)= \begin{cases}W_{R}-1-\frac{\lambda \lambda}{2}\left(W_{R}-i\right)\left(W_{R}-i+1\right) & \text { if } i \in\left[1, N-W_{R}\right] \\ N-1-\frac{\lambda \lambda}{2}\left[N(N+1)-2(N+1) i+2 i^{2}\right] & \text { if } i \in\left[N-W_{R}, N / 2\right]\end{cases}
$$


In summary, the overall profile of $\delta_{R}(i)$ is monotonically increasing until the middle and then monotonically decreasing, and in this sense is always a dome. Consider the load $f \leq 1$, then $N \lambda=1$ by definition and thus $\frac{1}{j \lambda} \geq N$. Thus for this case $\delta_{R}(i)=(N-1)-\frac{L \lambda}{2}[N(N+$ 1) $\left.-2(N-1) i+2 i^{2}\right]$; this expression will be used for most of the cases we subsequently discuss.

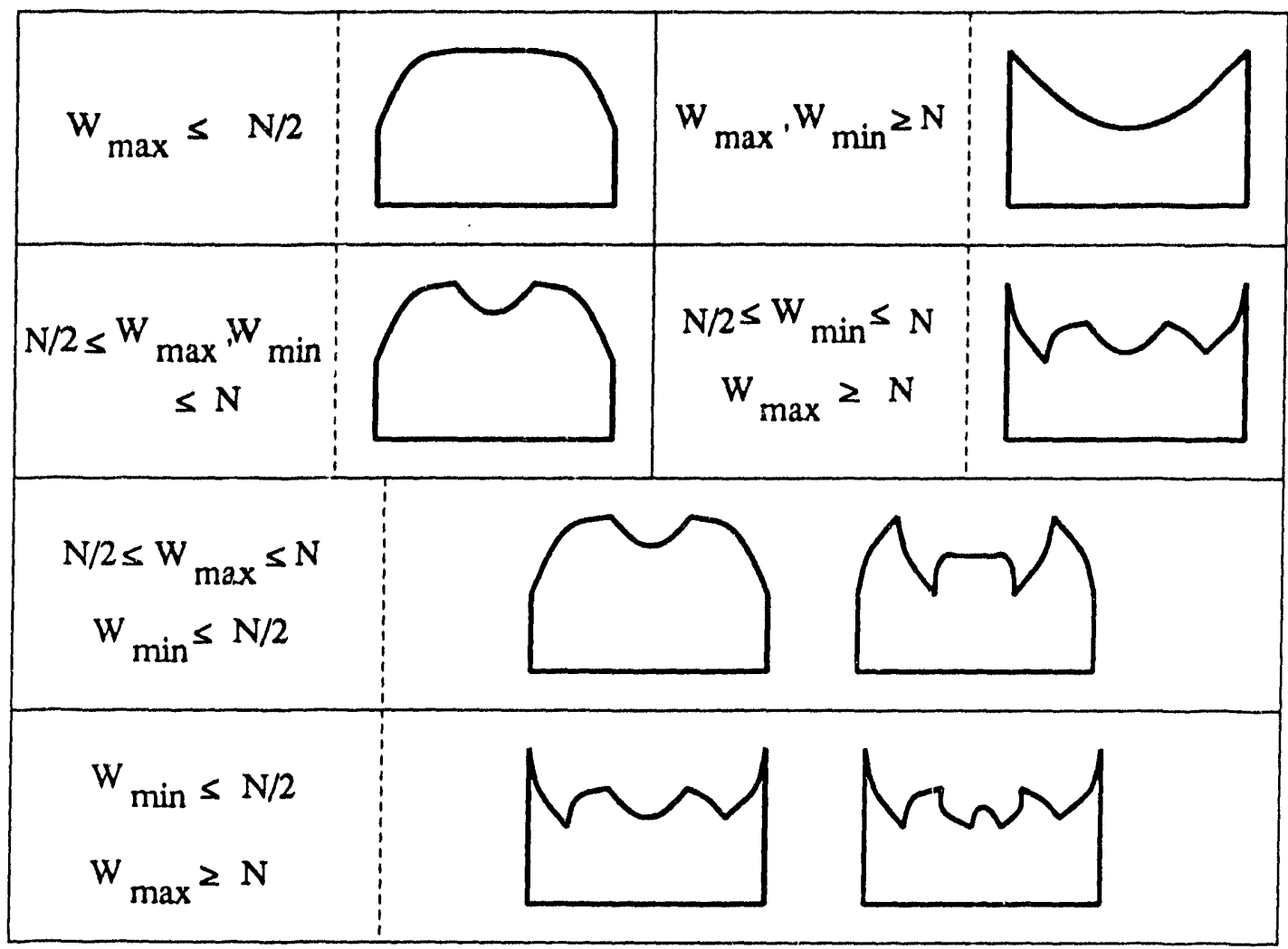

Figure 5: Summary of profiles of $W_{J}(i)$.

\subsection{Profiles of $\delta_{J}(i)$}

Based on the range of values for $W_{J}(i)$ we have eight basic profiles for $\delta_{J}(i)$ and their variants thereof as shown in Fig. 5. Since $W_{J}(i)$ depends on $i$, its analysis is not as straight forward as that of $\delta_{R}(i)$. To derive the profiles of $\delta_{J}(i)$, we first derive the expressions for the locus of $W_{J}(i)$ with the same value $x$. Let $\xi(i, x)$ denote the value of $\delta_{J}(i)$ when $W_{J}(i)=x$ such that $\delta_{J}(i)=\xi\left(i, W_{J}(i)\right)$. We then imagine a two-dimensional surface swept by $\xi(i, x)$ as we vary the values of $x$. This can be imagined in three-dimensions as a surface, and a suitable projection of the curve of $W_{J}(i)$ onto this surface yields the actual profile of the $\delta_{J}(i)$ as will be explained subsequently in this section.

We now consider three different cases for the constant waiting time locus $\xi(i, x)$. We have three basic profiles (see Appendix B for proofs) as shown in Fig. 6.

(a) $x \leq N / 2$ : The profile of $\xi(i, x)$ is monotonically increasing in the range $[1, x]$, is const ant in $[x, N-x]$ and is monotonically decreasing in the range $[N-x, N]$ as expressed in 


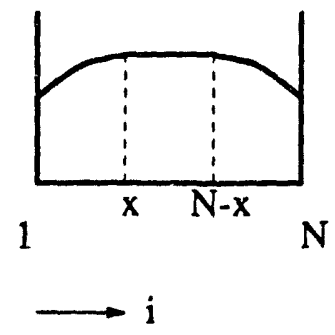

(a) $x \leq N / 2$

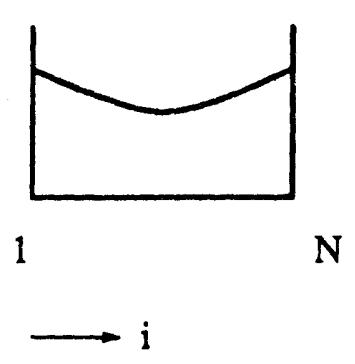

(b) $\mathrm{x}>\mathrm{N}$

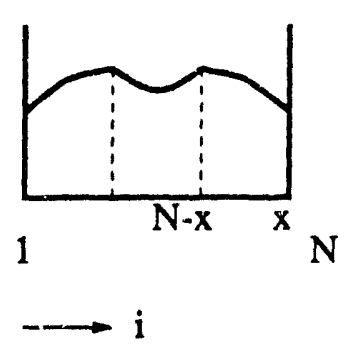

(c) $N / 2 \leq x<N$

Figure 6: Basic profiles of $\xi(i, x)$.

the following equation (Fig. 6(a)).

$$
\xi(i, x)= \begin{cases}f \lambda x(x-1) & \text { if } i \in[x, N / 2] \\ \frac{f \lambda}{2}[i(i-1)+x(x-1)] & \text { if } i \in[1, x]\end{cases}
$$

(b) $x \geq N$ : The profile is concave in that it is strictly decreasing until the middle and then is strictly increasing (Fig. 6(b)). Note that this behavior is reverse of that of $\delta_{R}(i)$ for case (b). The expression for $\xi(i, x)$ is given by

$$
\xi(i, x)=\frac{f \lambda}{2}\left[2 i^{2}-2 i(N+1)+N(N+1)\right]
$$

(c) $N / 2 \leq x \leq N$ : This is a combination of (a) and (b), where the profile is convex in the ranges $[1, N-x]$ and $[x, N]$, and is concave in range $[N-x, x]$ (Fig. 6(c)).

$$
\xi(i, x)= \begin{cases}\frac{\Delta \lambda}{2}[i(i-1)+x(x+1)] & \text { if } i \in[1, N-x] \\ \frac{\lambda \lambda}{2}\left[2 i^{2}-2 i(N+1)+N(N+1)\right] & \text { if } i \in[N-x, N / 2]\end{cases}
$$

Let $W_{\max }=\max _{i}\left\{W_{J}(i)\right\}$ and $W_{\min }=\min _{i}\left\{W_{J}(i)\right\}$. Now we derive the profiles of $\delta_{J}(i)$ by considering a three dimensional view of profiles of $\xi(i, x)$ as we vary values of $x$ from $W_{\min }$ to $W_{\max }$. Imagine that we first draw the profiles $W_{J}(i)$ as a function of $i$ in the plane with $\xi(i, x)=0$. Then we project this curve vertically onto the surface of $\xi(i, x)$ and read off the values corresponding to the projected curve (see Fig. 7).

We first consider three cases where the profiles of $\delta_{J}(i)$ are similar to the profiles of $\xi(i, x)$ discussed above.

(a) $W_{\max } \leq N / 2$ : Here the profile is increasing in the range $\left[1, W_{\max }\right]$, constant in the range $\left[W_{\max }, N-W_{\max }\right]$, and is decreasing in the range $\left[N-W_{\max }, N\right]$. Although this profile is similar to the case (a) above, the exact values and the derivatives are different in this case. Thus, from equation (4.2.1), we have

$$
\delta_{J}(i)= \begin{cases}f \lambda W_{J}(i)\left(W_{J}(i)-1\right) & \text { if } i \in\left[1, W_{\max }\right] \\ f \lambda\left[\frac{i(i-1)}{3}+\frac{W_{J}(i)\left(W_{J}(i)-1\right)}{2}\right] & \text { if } i \in\left[W_{\max }, N / 2\right]\end{cases}
$$

This profile can be inagined as a suitable cut in the three dimensional profile as shown in Fig. 7. Notice that the nodes with higher values for $W_{J}()$ are attained at nodes with higher $\delta_{J}()$ values. Thus no matter how the $W_{j}(i)$ s are distributed among the nodes, the profile is always convex or a dome. 


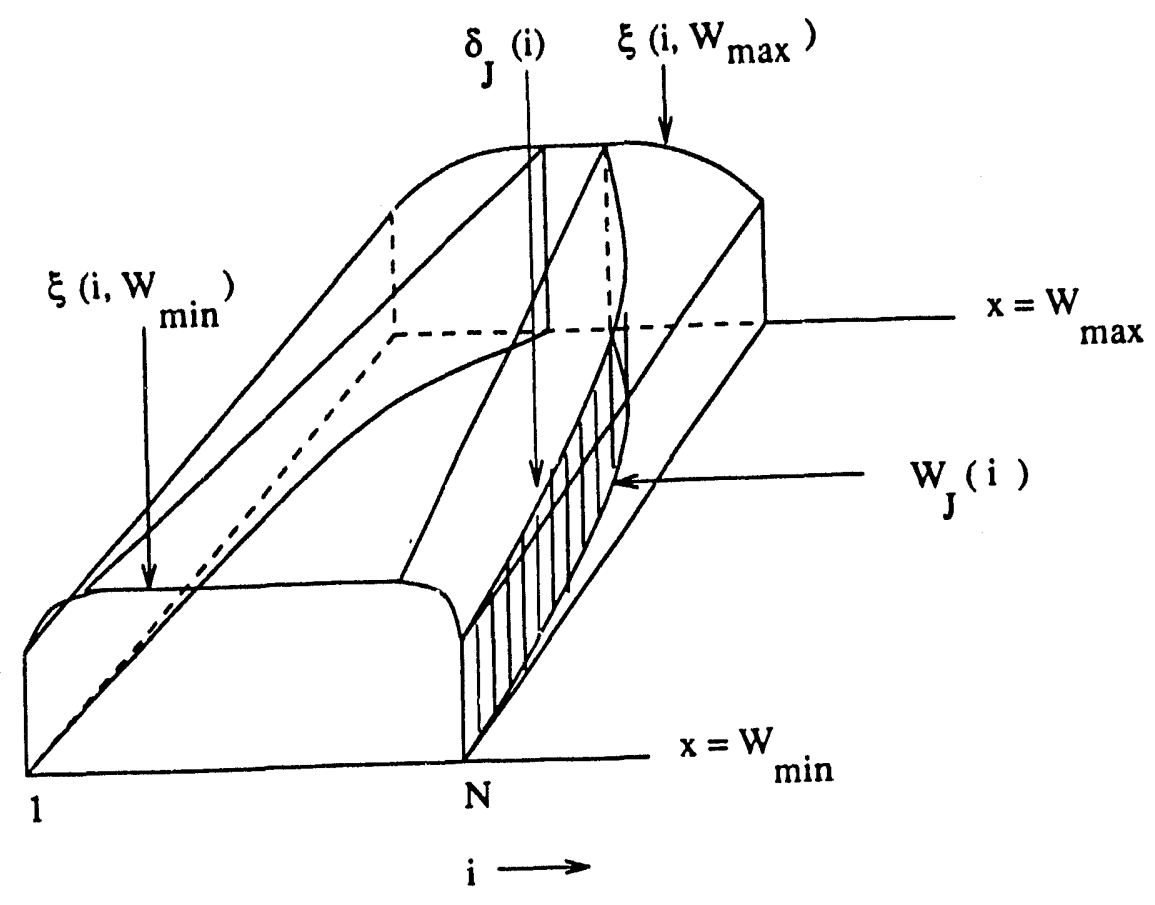

Figure 7: Profile of $\delta_{J}(i)$ for $W_{\max } \leq N / 2$.

(b) $W_{\min } \geq N$ : In this case $\delta_{J}(i)$ is monotonically decreasing until the middle and monotonically increasing from then on. This profile can be imagined as a suitable cut in the three dimensional profile as shown in Fig. 8. Notice that no matter how the $W_{j}(i) \mathrm{s}$ are distributed among the nodes, the profile is always concave or dome-up.

$$
\delta_{J}(i)=\frac{f \lambda}{2}\left[2 i^{2}-2 i(N+1)+N(N+1)\right]
$$

(c) $N / 2<W_{\min }, W_{\max }<N$ : This plot consists of three parts. Let $W_{\operatorname{mid}}=W_{J}(N / 2)$. We have $W_{\text {mid }} \geq W_{\min }$. The profile is as shown in Fig. 9, and the expression for $\delta_{J}(i)$ is as follows.

$$
\delta_{J}(i)= \begin{cases}f \lambda\left[\frac{i(i-1)}{2}+\frac{W_{J}(i)\left(W_{J}(i)-1\right)}{2}\right] & \text { if } i \in\left[1, N-W_{\max }\right] \\ \frac{f \lambda}{2}\left[2 i^{2}-2 i(N+1)+N(N+1)\right] & \text { if } i \in\left[N-W_{\max }, N / 2\right]\end{cases}
$$

In this case, the profile is increasing in the interval $\left[1, N-W_{\max }\right]$, concave in the interval $\left[N-W_{\max }, W_{\max }\right]$, and is decreasing in the interval $\left[W_{\max }, N\right]$.

Now we consider the other three cases where the profile does not belong to one of the basic profiles of equations (4.2.1) through (4.2.3). In each of the following case, several variants are possible. These variants are combinations of the basic profiles discussed above. For each case, we only discuss typical variants, and other variants can be derived similarly. 


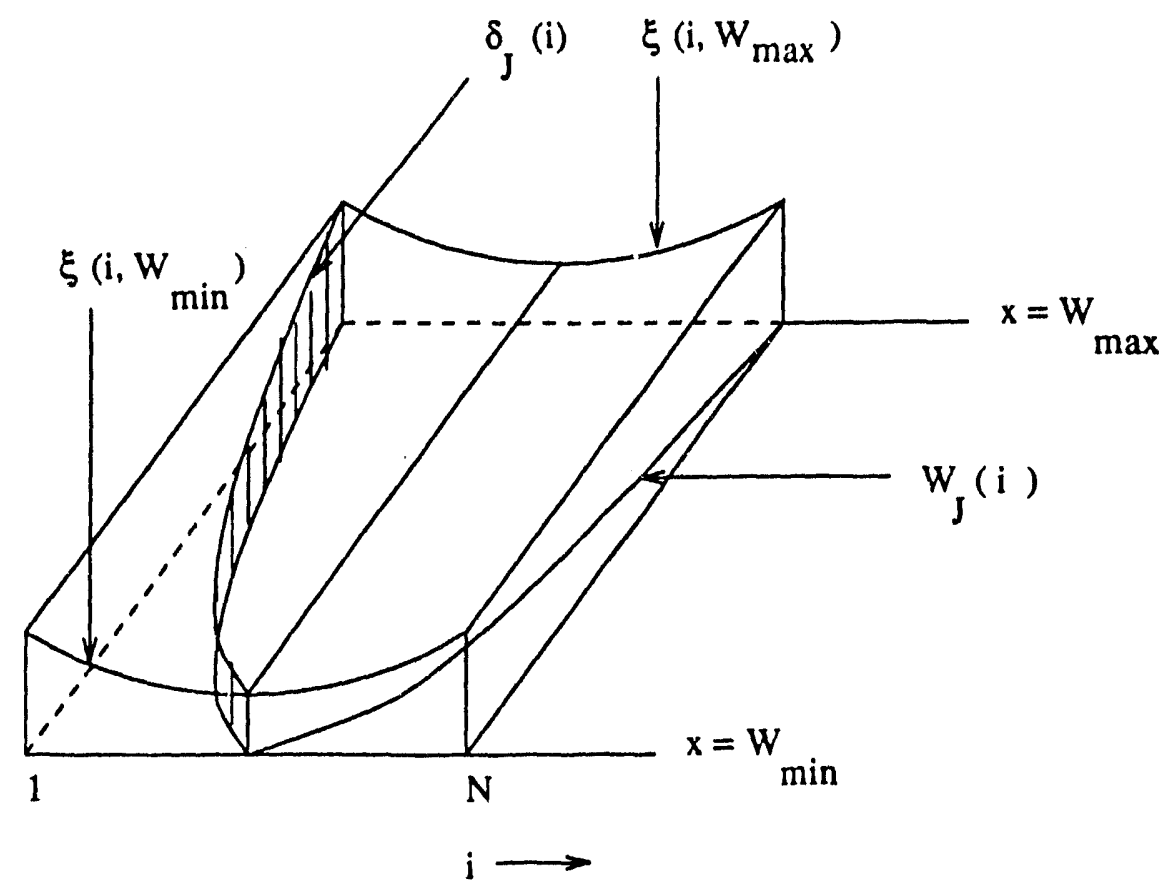

Figure 8: Profile of $\delta_{J}(i)$ for $W_{\min }, W_{\max } \geq N$.

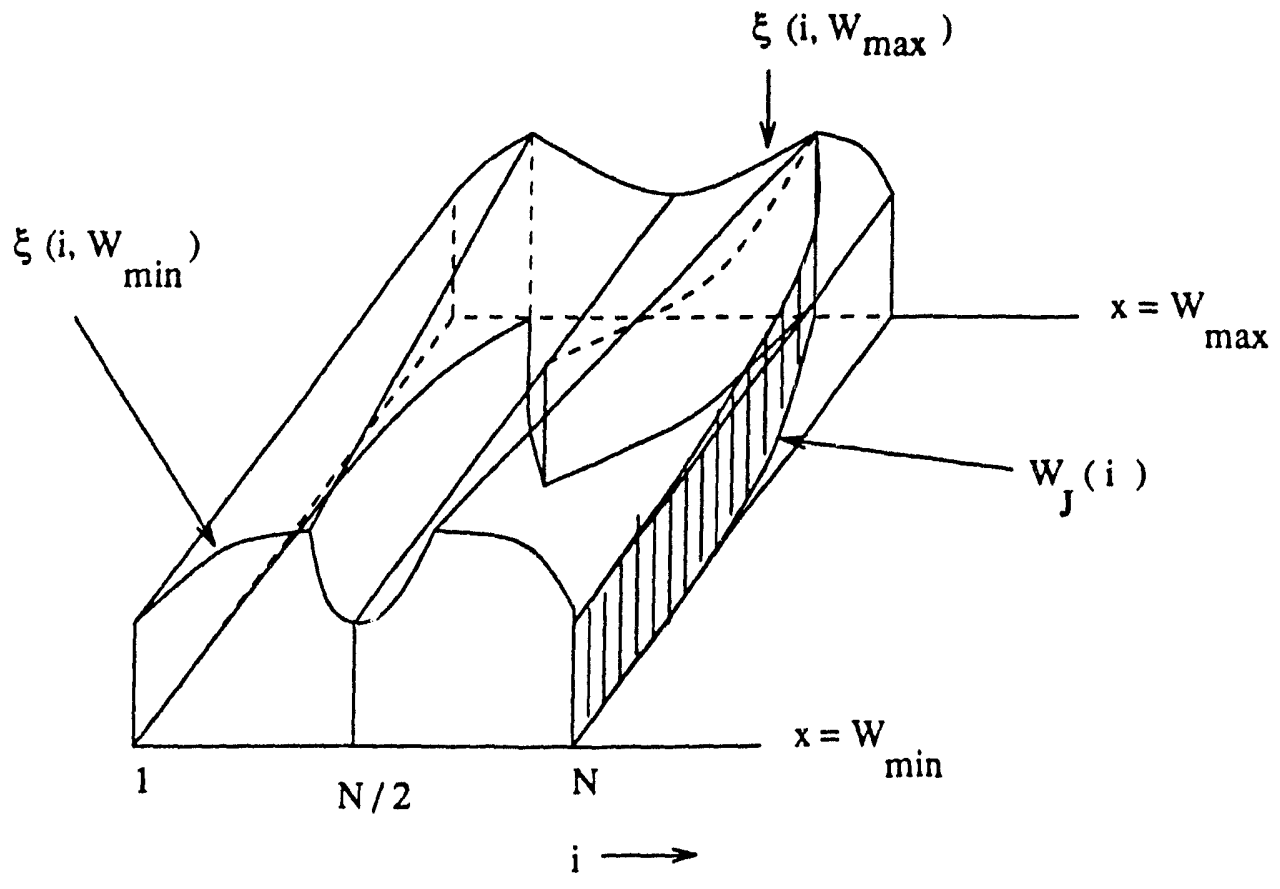

Figure 9: Profile of $\delta_{J}(i)$ for $N / 2 \leq W_{\max }, W_{\min } \leq N$. 


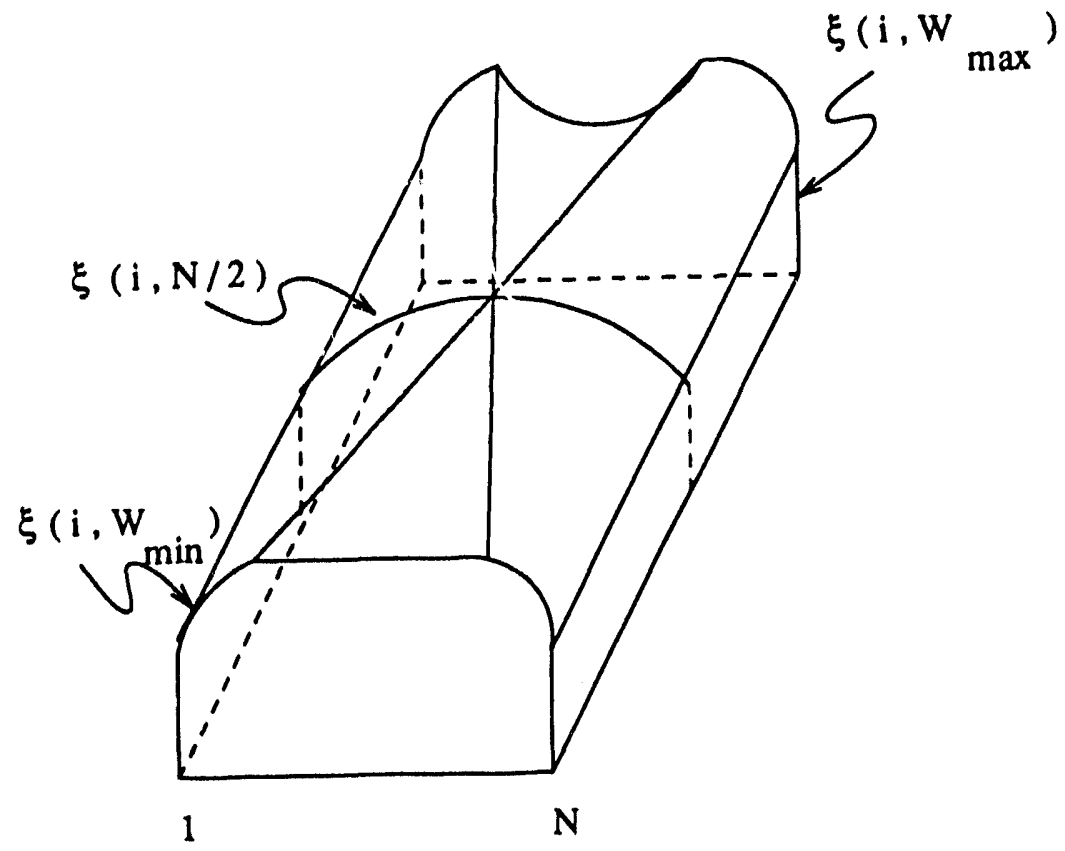

(a) $\xi(i,$.
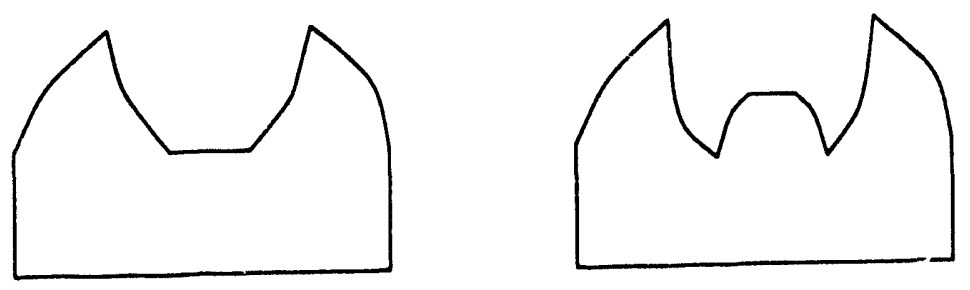
(b) $\mathrm{W}_{\text {mid }}>\mathrm{N} / 2$
(c) $\mathrm{W}_{\text {mid }}<\mathrm{N} / 2$

Figure 10: Profile of $\delta_{J}(i)$ for $W_{\min } \leq N / 2$ and $N / 2 \leq W_{\max } \leq N$.

(d) $W_{\min }<N / 2$ and $N / 2<W_{\max }<N$ : Let $N / 2=W_{J}\left(I_{N / 2}\right)$ for some $I_{N / 2} \in[N-$ $\left.W_{\max }, W_{\max }\right]$. If $W_{\text {mid }}<N / 2$ then we have the following profile: the profile is increasing in the interval $\left[1, N-W_{\max }\right]$, decreasing in $\left[N-W_{\max }, I_{N / 2}\right]$, and is similar to case (a) in the interval $\left[I_{N / 2}, N / 2\right]$ (see Fig. $10(\mathrm{c})$ ). If $W_{\text {mid }}>N / 2$, the profile is increasing in the range $\left[1, N-W_{\max }\right]$ and is decreasing in the interval $\left[N-W_{\max }, N / 2\right]$ (see Fig. $10(\mathrm{~b}))$. In the middle portion, the plot is concave as in Case (c).

(e) $W_{\min }<N / 2$ and $W_{\max }>N$ : This case generates most varied profiles. There are two variants of this profile as shown in Fig.11. 


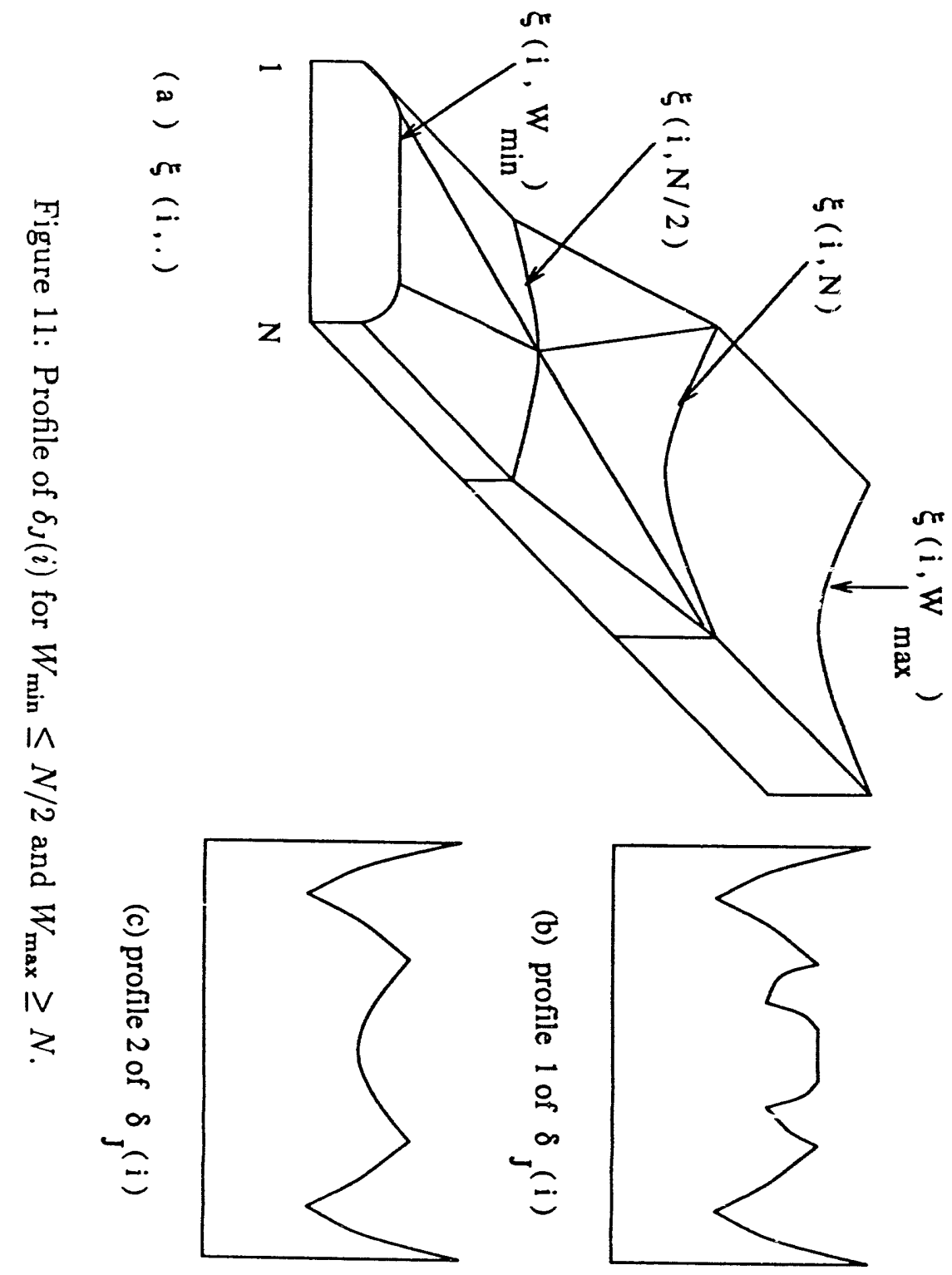


(f) $N / 2<W_{\min }<N$ and $W_{\max }>N$ : The profile of this case is shown in Fig. 12 .
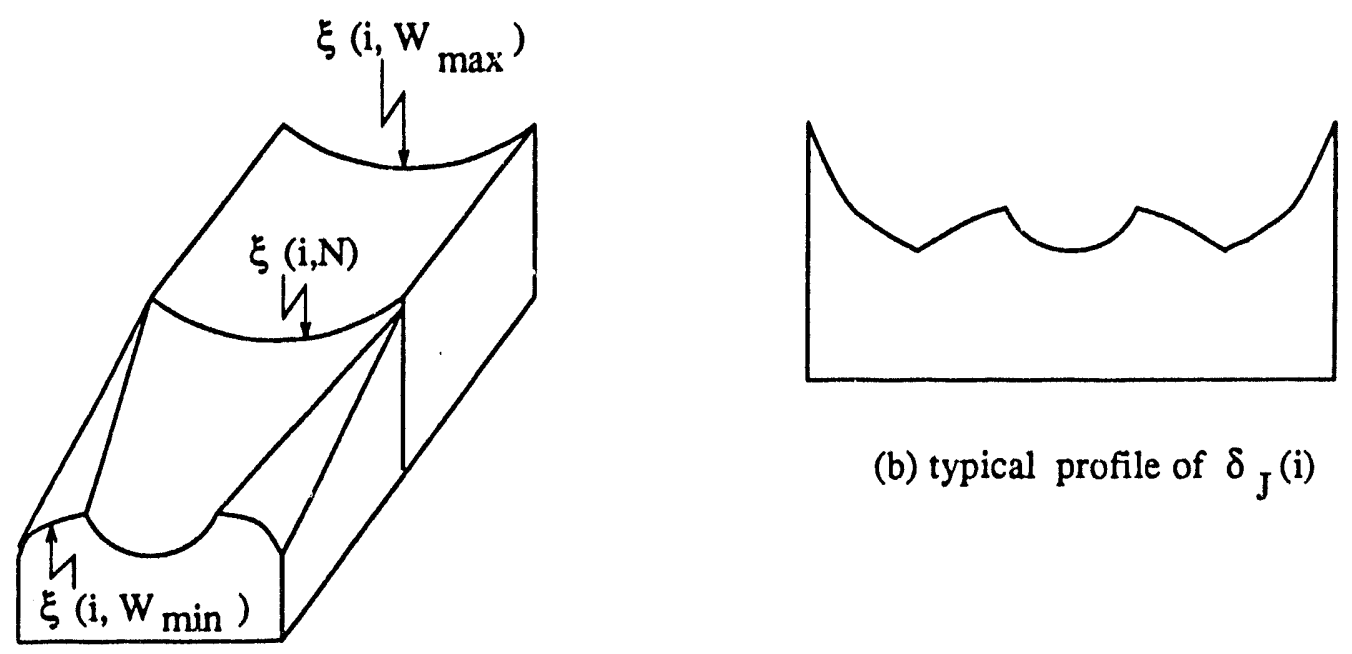

(b) typical profile of $\delta_{\mathrm{J}}$ (i)

(a) $\xi(i,$.

Figure 12: Profile of $\delta_{J}(i)$ for $N / 2 \leq W_{\min } \leq N / 2$ and $W_{\max } \geq N$.

Because of the interaction between the $W(i)$ and $\delta_{J}(i)$, it is not direct to quantify the profiles of $\delta_{J}(i)$ as in the case of $\delta_{R}(i)$. As in the above cases, the profile in general can be categorized into one of the basic categories by suitably considering the intervals for $i$. Within each identified interval we can directly obtain ihe formulae for $\delta_{J}(i)$ from the basic equations of (4.2.1) through (4.2.3). In Case (e) we have the most number of intervals which is nine. In the extreme cases, we only have a single interval for $i$ such that the profile can be easily expressed as one of the basic types.

\subsection{Waiting time Profiles}

Since there are three distinct profiles for $\delta_{R}(i)$ and eight for $\delta_{J}(i)$, in all we conceivably have at least twenty four possible profiles for $\Delta(i)=\delta_{R}(i)+\delta_{J}(i)$. In this section, we discuss only four cases that include those obtained in our simulation and reported by others. The summary of this section is shown in Fig. 2, where the evolution of the waiting time profiles with the increase in the load is shown. Notice the transition, where the profiles start out being convex and develop a concavity in the middle as the load is increased. As we approach high loads, the concavity expands and occupies the the entire range of the profile.

The profiles of $\delta_{J}(i)$ are expressed as functions of $W_{J}(i)$, and hence it is not directly possible to conclude the profiles of the waiting times from the values of $\Delta(i)$. In the next subsection, we consider approximating the relationship between $\Delta(i)$ and $W_{J}(i)$ so that we will be able to eliminate $\Delta(i)$ from the expressions for $W_{J}(i)$. The outline of our method is as follows. We show that $W_{J}(i)$ satisfies one of the following types of equations in the appropriate ranges for $i$.

$$
\left[W_{J}(i)-1\right]^{2}=X(i)+A W_{J}(i)\left[W_{J}(i)+1\right]
$$




$$
\left\{W_{J}(i)-1\right\}^{2}=X(i)+A
$$

where $W_{J}(i)$ and $X(i)$ are functions of $i$ and $A$ does not depend $o n i$. In the Appendix D we show that the bounds of $W_{J}(i)$ can be expressed as follows:

$$
\begin{aligned}
& W_{J}(i) \leq B X(i)+C \\
& W_{J}(i) \geq D X(i)+E
\end{aligned}
$$

where $B, C, D$ and $E$ do not depend on $i$. Thus, we have $W_{J}(i)=\Theta(X(i))$, where $X(i)$ is derived from the expression for $\Delta(i)$. In other words, we show that both $\Delta(i)$ and $W_{J}(i)$ have same profiles within constants that do not depend on $i$.

\subsubsection{Expected Waiting tin:e}

Since $\Delta(i)$ is a measure of impedance that a job at node $i$ faces, it gives a measure of the probability that the job will not be delivered in the present slot. Then we approximate $c \Delta(i)$ to be the probability that the present job does not get delivered in the next slot, where $c$ is the normalization constant. Let $1-c \Delta(i)$ be the probability that the present job is delivered in the present slot. Now, the expected number of slots that the present job waits is given by

$$
W_{J}(i)=\sum_{i=1}^{\infty} i[c \Delta(i)]^{i-1}[1-c \Delta(i)]
$$

After some algebraic manipulation, we can show that

$$
W_{J}(i)=\frac{1}{1-c \Delta(i)}=\left[1+c \Delta(i)+(c \Delta(i))^{2}+\ldots\right] .
$$

Consider the following approximation (that assumes $(c \Delta(i))^{2} \approx 0$ )

$$
W_{J}(i) \approx 1+c \Delta(i)
$$

which shows a linear relation between $\Delta(i)$ and $W_{J}(i)$. On the other hand, the equations of Section 4.2. show a quadratic relation. Thus more appropriate approximation would be to assume that $\sqrt{c \Delta(i)}$ to be the probability that a present job gets delivered in the next slot, and by proceeding in the above manner, we obtain $W_{J}(i)=1+\sqrt{c \Delta(i)}$. Thus we have

$$
\Delta(i)=\left[W_{J}(i)-1\right]^{2} / c .
$$

We will subsequently show that $\Delta(i)$ has a form $X(i)+A W_{J}(i)\left[W_{J}(i)+1\right]$ or $X(i)+A$, where $A$ is independent of $i$. Thus by combining Eq. (4.3.1.2) with these forms and using Eq. (4.3.3) and $\mathrm{Eq}$ (4.3.4), we conclude

$$
W_{J}(i)=\Theta[\Delta(i)] .
$$

This important result establishes that both $\Delta(i)$ and $W_{J}(i)$ have the same profiles when viewed as functions of $i$. 


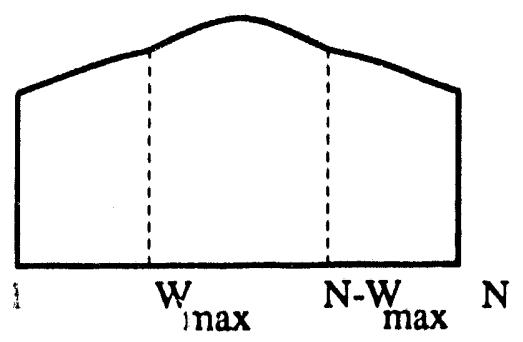

(a) $\mathrm{W}_{\mathrm{R}} \geq \mathrm{N}$ and $\mathrm{W}_{\max } \leq \mathrm{N} / 2$

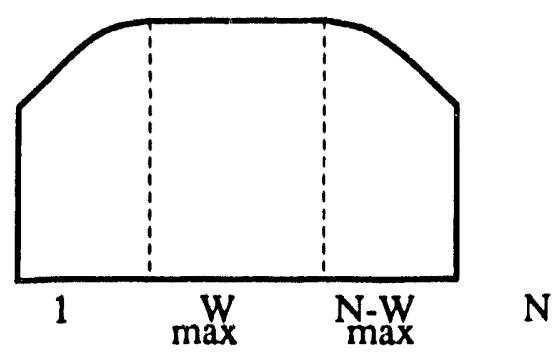

(b) $\mathrm{W}_{\mathrm{R}} \geq \mathrm{N}$ and $\mathrm{N} / 2 \leq \mathrm{W}_{\min }, \mathrm{W}_{\max } \leq \mathrm{N} / 2$

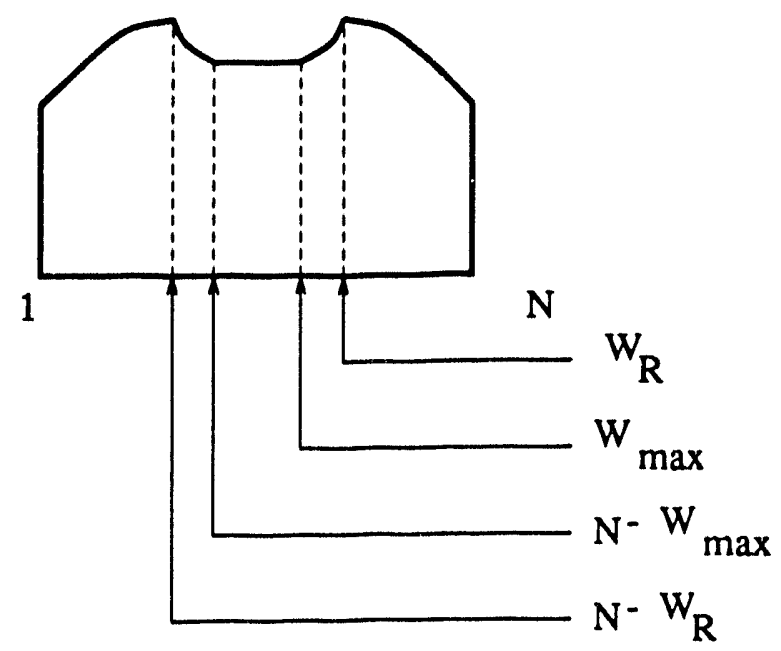

(c) high to overload

Figure 13: Profiles of $\Delta(i), W_{J}(i)$ for low-to-medium, medium-to-high an high-to-overload.

\subsubsection{Low to Medium Loads}

We consider the loads such that $W_{\max }<N / 2$ and $W_{R} \geq N$. The impedance factor $\Delta(i)=$ $\delta_{J}(i)+\delta_{R}(i)$ is given by

$$
\Delta(i)= \begin{cases}(N-1)+f \lambda\left[W_{J}(i)\left(W_{J}(i)-1\right)-\frac{N^{2}+N}{2}+(N+1) i-i^{2}\right] & \text { if } i \in\left[1, W_{\max }\right] \\ (N-1)+\frac{\hbar \lambda}{2}\left[\frac{W_{J}(i)\left(W_{J}(i)-1\right)}{2}-N^{2}-N+2 N i+i-i^{2}\right] & \text { if } i \in\left[W_{\max }, N / 2\right]\end{cases}
$$

Profile of $\Delta(i)$ is shown in Fig. 13(a), which is convex in all the three possible ranges of $i$. As functions of $i, W_{J}(i)=\Theta[\Delta(i)]$ and hence the profile of $W_{J}(i)$ is also as shown in Fig. 13(a). However, it must be noted the actual values of these functions could be quite different. Now using Equation (4.3.1.2) and Appendix D, we can express the $W_{J}(i)$ as follows.

$$
W_{J}(i)= \begin{cases}\Theta\left[(N-1)+\frac{f \lambda}{2}\left[-N(N+1)+2(N+1) i-2 i^{2}\right]\right. & \text { if } i \in\left[1, W_{\max }\right] \\ \Theta\left[(N-1)+\frac{\perp \lambda}{2}\left[-N^{2}+N+i-i^{2}\right]\right] & \text { if } i \in\left[W_{\max }, N / 2\right]\end{cases}
$$




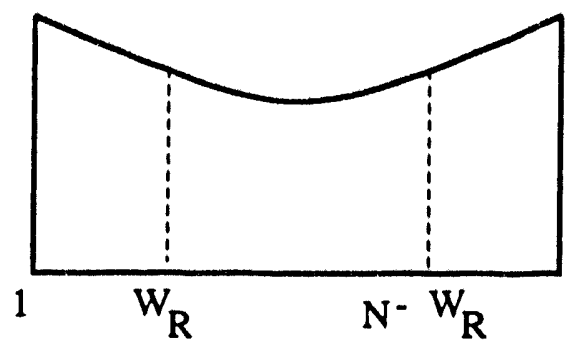

(a) $\mathrm{W}_{\mathrm{R}} \leq \mathrm{N} / 3$

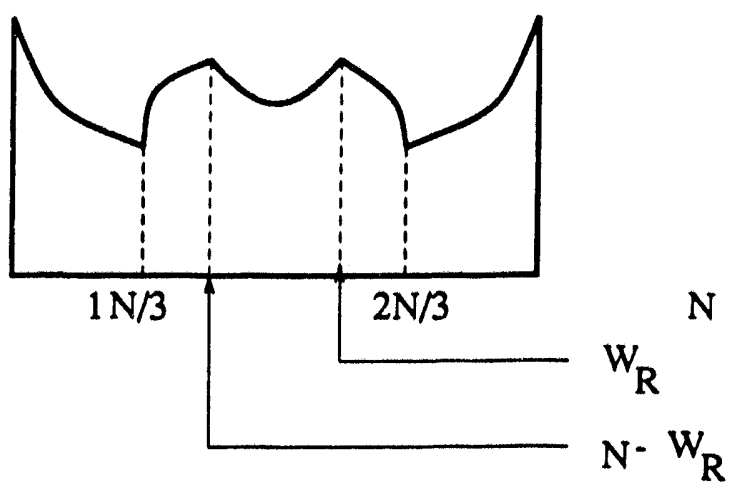

$N B S W_{R} \leq N / 2$

Figure 14: Profiles of $\Delta(i), W_{J}(i)$ for overload.

This predicted shape closely matches our simulation results in Fig. 15. Also it closely matches the shapes based on simulation results presented by other authors, for example Fig. 8 of [17].

\subsubsection{Overload}

We consider the loads where $W_{\min } \geq N$ and $W_{R}(i) \leq N / 2$. The other cases for different ranges of $W_{R}(i)$ can be similarly obtained. Thus we have

$$
\Delta(i)= \begin{cases}\frac{1}{\lambda f}-1+\frac{\lambda \lambda}{2}\left[2 i^{2}-2 i(N+1)+N(N+1)\right] & \text { if } i \in\left[W_{R}, N / 2\right] \\ \frac{1}{2 \lambda f}-\frac{3}{2}+\frac{\ell \lambda}{2}\left[i^{2}+i\left(\frac{2}{\lambda f}-2 N-1\right)+N(N+1)\right] & \text { if } i \in\left[1, W_{R}\right]\end{cases}
$$

The profile of $\Delta(i)$ depends on the value of $W_{R}=\frac{1}{\lambda f}$. In general the over all behavior of $\Delta(i)$ is concave, but can have some non-concave profiles in some range. This function is definitely concave in the middle region $\left[W_{R}, N-W_{R}\right]$, and also in the end regions $[1, N / 3]$ and $[2 / 3 N, N]$. If $W_{R} \leq N / 3$, which happens under overload, then the entire profile is concave as shown i.2 Fig. 14(a). In this case the profiles of $W_{J}(i)$ is given by

$$
W_{J}(i)= \begin{cases}\Theta\left[\frac{\Delta \lambda}{2}\left[2 i^{2}-2 i(N+1)+N(N+1)\right]\right] & \text { if } i \in\left[W_{R}, N / 2\right] \\ \Theta\left[\frac{\delta \lambda}{2}\left[i^{2}+i\left(\frac{2}{\lambda J}-2 N-1\right)+N(N+1)\right]\right] & \text { if } i \in\left[1, W_{R}\right]\end{cases}
$$

The profiles of $W_{J}(i)$ for the cases of $W_{R} \leq N / 3$ and $N / 3<W_{R}(i) \leq N / 2$ are shown in Fig. 14 (a) and (b) respectively. The shape of Fig. 14 (a) has been observed during the simulation under overload as in Fig. 15.

\subsubsection{Medium to High Loads}

In this case we consider that $f \leq 1$. We present the case $N / 2 \leq W_{\min }, W_{\max } \leq N$ and $W_{R} \geq N$. In this case we have.

$\Delta(i)= \begin{cases}(N-1)+\frac{f \lambda}{2}\left[W_{J}(i)\left(W_{J}(i)+1\right)-N(N+1)-i^{2}+2 N i+i\right] & \text { if } i \in\left[1, W_{\max }\right] \\ (N-1) & \text { if } i \in\left[W_{\max }, N / 2\right]\end{cases}$ 


$$
W_{J}(i)= \begin{cases}\Theta\left[2 N i+i-N(N+1)-i^{2}\right] & \text { if } i \in\left[1, W_{\max }\right] \\ \Theta(1) & \text { if } i \in\left[W_{\max }, N / 2\right]\end{cases}
$$

The profiles of this case are shown in Fig. 13(b). Note that the profiles are convex, which are observed in simulation (see Fig. 15(a)).

\subsubsection{High to Overload}

In this case we consider that $f \geq 1, N / 2 \leq W_{\min }, W_{\max } \leq N$ and $N / 2 \leq W_{R} \leq N$. In this case we have two separate cas ss (a) $W_{R} \leq W_{\max }$ and (b) $W_{R}>W_{\max }$. We consider only the first case, and the other case is similar.

$$
\Delta(i)= \begin{cases}\left(\frac{1}{\lambda f}-1\right)+\frac{L \lambda}{2}\left[2 i^{2}-2 i(N+1)+N(N+1)\right] & \text { if } i \in\left[1, W_{R}\right] \\ \left(\frac{1}{\lambda f}+1\right)+\frac{L \lambda}{2}\left[3 i^{2}-i\left(\frac{2}{\lambda f}+3+2 N\right)+N^{2}+N\right] & \text { if } i \in\left[W_{R}-W_{\max }\right] \\ N-1 & \text { if } i \in\left[W_{\max }-N / 2\right]\end{cases}
$$

The profiles for $W_{J}(i)$ can be obtained as follows (see Fig. 13(c)):

$$
W_{J}(i)= \begin{cases}\Theta\left[\frac{\perp \lambda}{2}\left[2 i^{2}-2 i(N+1)+N(N+1)\right]\right] & \text { if } i \in\left[1, W_{R}\right] \\ \Theta\left[\frac{\perp \lambda}{2}\left[3 i^{2}-i\left(\frac{2}{\lambda f}+3+2 N\right)+N^{2}+N\right]\right] & \text { if } i \in\left[W_{R}-W_{\max }\right] \\ \Theta(1) & \text { if } i \in\left[W_{\max }-N / 2\right]\end{cases}
$$

Recall the summary in Fig. 2, which illustrates how the waiting time profiles evolve with the increase in the load. The profile starts out being convex at low loads and becomes concave as the load reaches high values. In the transition, the profiles exhibit behavior that can be captured by a finite combination of basic profiles.

\section{Simulation Model}

The sinsulation model is based on the discrete event simulation view and is written in Simscript. Because simulation runs tend to be lengthy we have modeled only one bus at $150 \mathrm{Mb} / \mathrm{s}$ and extrapolated to the full two-bus system by taking weighted averages of the results.

In the validation phase we calculated the metrics discussed below for each subinterval of $50 \mathrm{~ms}$ of simulation time as well as for the entire simulation. Using a $90 \%$ confidence threshold we stopped the simulation when the confidence interval was smaller than $10 \%$ of the metrics calculated for the entire simulation time and the metric value was within the interval. We determined this time for a variety of parameter choices and established that in all stable network situations two seconds of simulation time was onough to meet the above criterion. We ran each case with up to five different random number streams and when run for the proper time obtained results within less than $5 \%$ of each other. The one exception is explained in the section on near chaotic performance when this behavior was not shown.

We studied variations on parameters such as network length, network bandwidth, number of nodes, message size and offered load and metrics ranging from throughput, wait time, response time and service time. At this point of our study we made the assumption that traffic was uniformly distributed both in regard to source and destination. In this paper, 


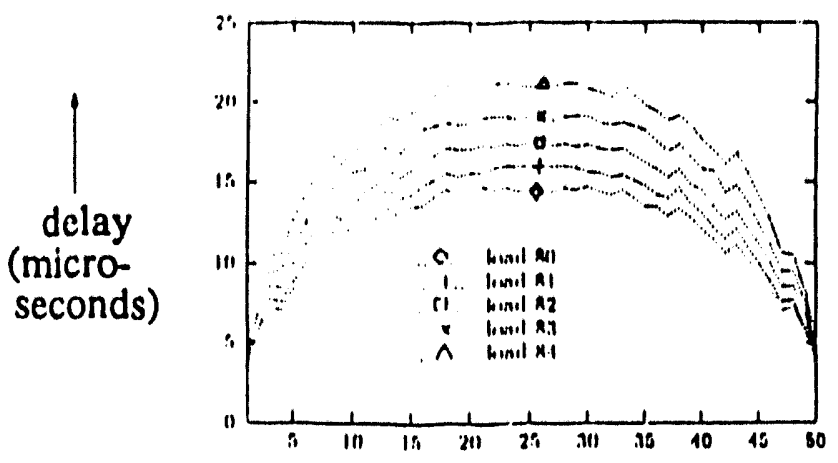

node number

(a) $80-84 \%$

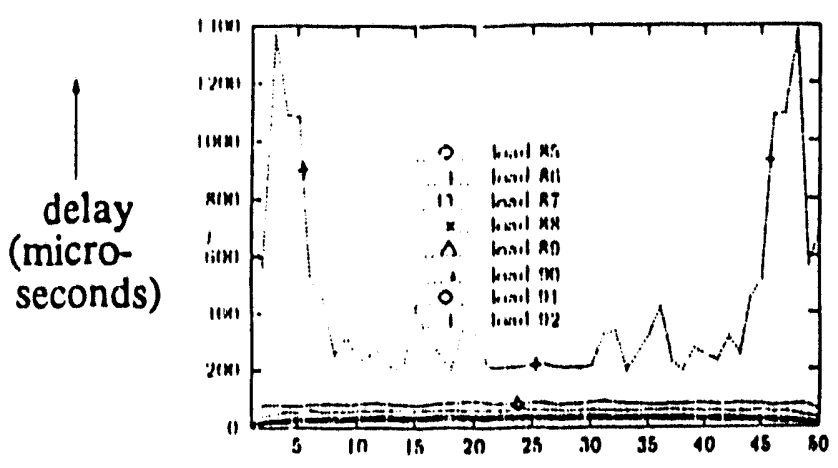

node number

(c) $85-92 \%$

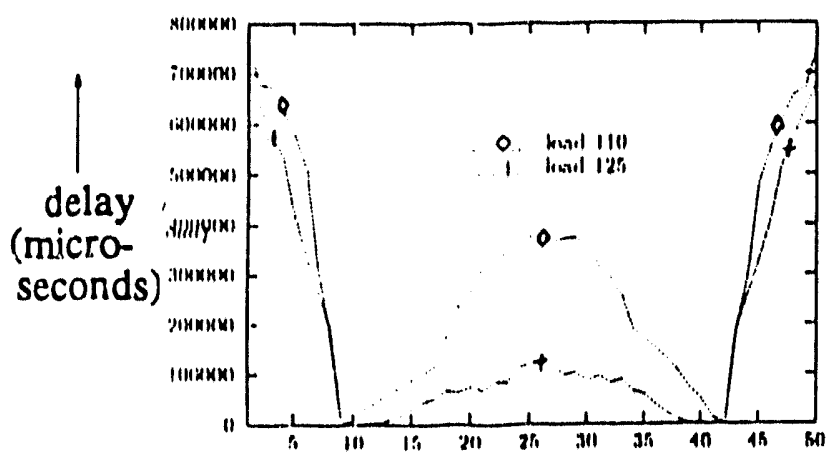

node number

(e) $93-100 \%$
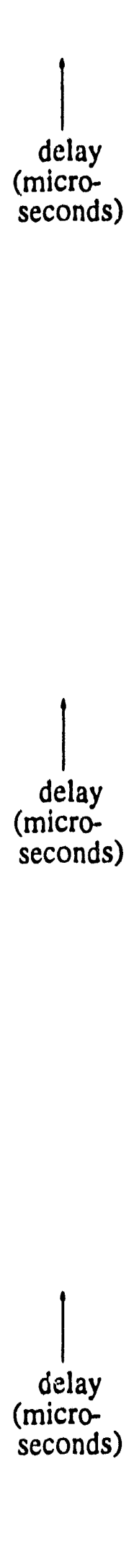

Figure 15: Simulation results at various loads.

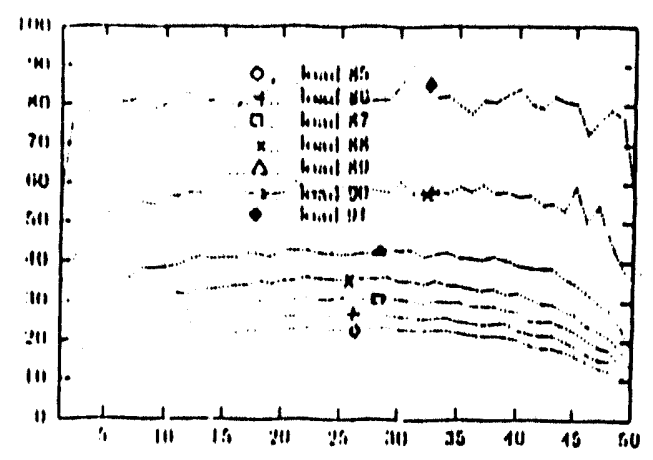

node number

(b) $85-93 \%$

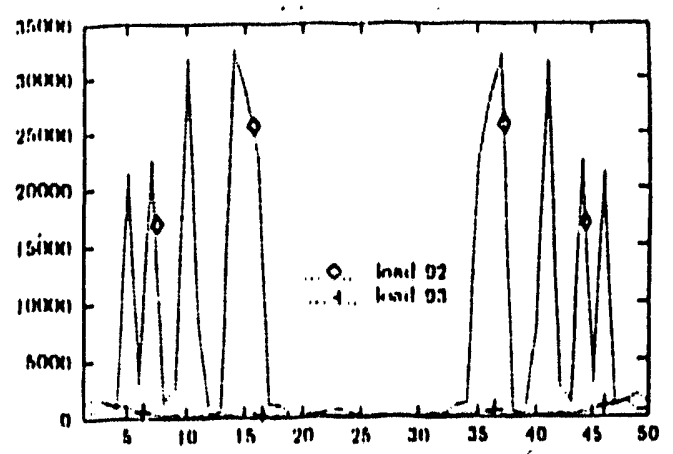

node number

(d) $92-93 \%$

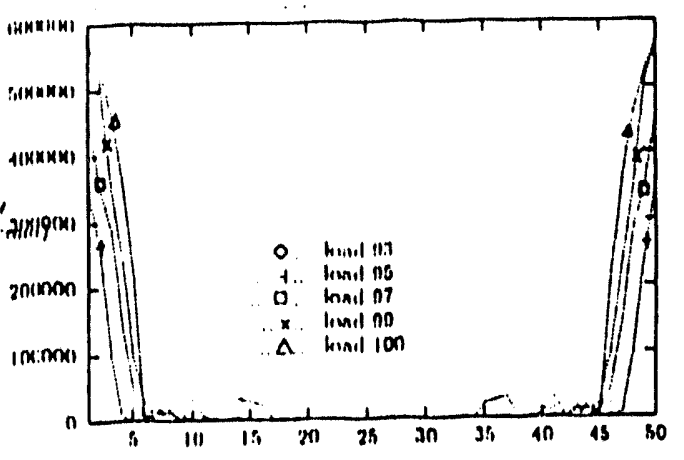

node number

(f) $110 \%, 125 \%$ 
though, we restrict our attention to the metrics of waiting time and throughput and have selected the case of a $50 \mathrm{~km}$ network with 50 equally spaced nodes. We varied offered load from $0 \%-125 \%$ and it should be observed that an offered load of $92.3 \%$ corresponds nominally to a full bus because of the 24 bits added by DQDB to each segment sent.

Our model can handle any message size and one variation allows for full ordering of segments and messages across the network by recording the status of the request bit counter for each segment as it arrives. Since most extant studies concentrate on the segment level we confine our study to messages of the size of a segment.

\section{Semblance of Chaos}

It has been observed during simulations that around a critical load, the system exhibited behavior akin to chaos, where no particular profile was observed. This behavior is explained by using an approximate analysis coarser than that in last section. More specifically, we show that a small change in the load causes the system to switch from convex to concave profiles and vice versa.

We characterize the DQDB by the average waiting time $\bar{W}$, such that no job takes more than $\bar{W}$ units of time to get delivered when averaged over all nodes, i.e., $\bar{W}=\frac{1}{N} \sum_{i=1}^{N} W_{J}(i)$. When viewed in this simplified framework, it suffices to consider the oncoming jobs that have been received in the $\bar{W}$ units of time (in terms of slots). We estimate $\bar{W}$ for the job by estimating a suitable parameter $W_{r}$ (different from $W_{R}$ ) for the request bits. Imagine that we are waiting to put a request bit on the network in the down stream traffic. The probability that we will be able to put it in time period $T$ can be approximated by $(1-f) n \lambda T /(n \lambda T)=$ $(1-f)$ which is the ratio of the number of free-slots encountered to the total number of slots encountered. Thus the expected number of slots that are required to put a request bit on the down traffic is given by $W_{r}=\sum_{i=1}^{\infty} i f^{i-1}(1-f)$ where the term inside the summation symbol corresponds to the probability that the request bit is delivered in the $i$ th slot. Now we have $S=\sum_{i=0}^{\infty} f^{i}=\frac{1}{1-f}$. By noting that $\frac{\partial S}{\partial f}=\sum_{i=1}^{\infty} i f^{i-1}=\frac{1}{(1-f)^{2}}$, we obtain $W_{r}=\frac{1}{1-f}$.

Now consider the "opposition" faced by a request bit when we attempt to put it on the upstream. On the average, we need not consider the request bits that arrive after $W_{r}$ slots since our request bit must have been gone by then. Hence it suffices to look at the request bits that are generated at the stations downstream within the period of $W_{r}$ slots. Thus the jobs that "oppose" our reservation bit are the ones that arrive at station $i$ within $W_{r}$ units from the current time. These are the jobs that will be generated in the time interval $\left[t, t+W_{r}-1\right]$ at station $i+1$, in the time interval $\left[t, t+W_{r}-2\right]$ at station $i+2$, and so on.

Compared to a request bit, a job faces two types of "opposition": (a) due to the jobs that arrive at nodes upstream before the present request propagates to them (b) request bits that have been accumulated. Since the "opposition" faced by a job is, on the average, "approximately twice" as much as that faced by a request bit, we have $\bar{W}=2 W_{r}=\frac{2}{1-f}$. Although this is a very coarse approximation, it predicts the shapes very closely. As in the case of $\delta_{R}(i)$, we will have different "impedances" for different nodes, even though the average value of $\bar{W}$ is the same throughout. Here we have two extreme basic cases (the 


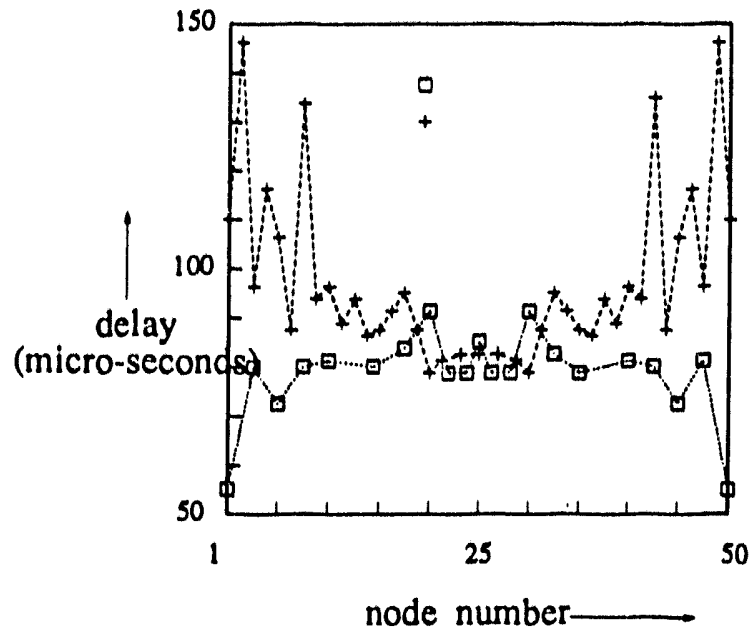

(a) load $91 \%$

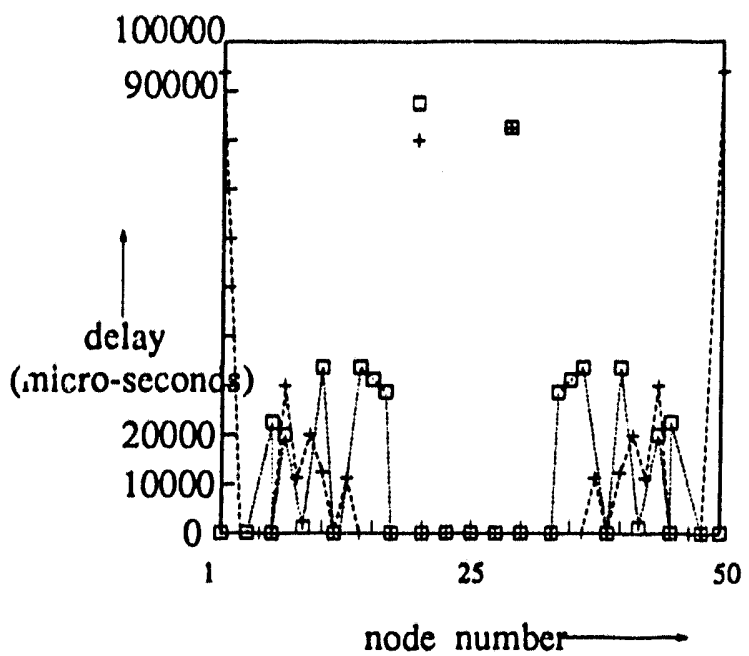

(b) load $93 \%$

Figure 16: Simulation results indicating semblance of chaos.

actual derivation which is a simplified version of that of last section, is given in Appendix E):

(a) Low-Load: Here $\bar{W} \leq N / 2$ or $f \leq 1-4 / N$; the profile is convex given by:

$$
\Delta(i)= \begin{cases}f \lambda \bar{W}(\bar{W}-1) & \text { if } i \in[\bar{W}, N / 2] \\ f \lambda\left[\frac{i(i-1)}{2}+\frac{\bar{W}(\bar{W}-1)}{2}\right] & \text { if } i \in[\bar{W}, N / 2]\end{cases}
$$

(b) High-Load: Here $\bar{W}>N$ or $f \geq 1-2 / N$; the profile is concave given by:

$$
\Delta(i)=\frac{f \lambda}{2}\left[2 i^{2}-2(N+1) i+N(N+1)\right] .
$$

Consider the critical load where system transits from the state for which $\bar{W}=N / 2$ to $\bar{W}=N$. Let $f_{N}$ and $f_{N / 2}$ denote the load corresponding to the conditions $\bar{W}=N$ and $\bar{W}=N / 2$ respectively. When $\bar{W}$ varies from $N / 2$ to $N$ the behavior of the system changes from one extreme to the other. Consider the change of load that is required to cause this reversal of behavior. Note that $N / 2=\frac{2}{1-f_{N / 2}}$ and $N=\frac{2}{1-f_{N}}$. A simple calculation shows that $\Delta f=f_{N}-f_{N / 2}=\frac{2}{N}$ This equation indicates that for bigger values of $N$ even infinitesimally small fluctuation in load causes the system to swing from one extremely to the other. This is particularly interesting because the arrival of suitably interleaved jobs could make the system switch back and forth. Technically speaking there is a difference in this type of behavior and chaos in non-linear system [32] because of the probabilistic characterization of our system. Experimental (simulation) evidence of this phenomenon is found in erratic behavior of the network around the critical load as shown in Fig. 16. For this system $N=50, f_{N / 2}=0.92$, and $f_{N}=0.96$. In each figure, we show results corresponding to two different random number generators. 


\section{General DQDB Model}

Now consider the case where the distance between the adjacent nodes is not uniform. Then we can visualize ${ }^{5}$ parameters $y_{i j}^{L}, y_{i j}^{R}, z_{i j}^{L}$ and $z_{i j}^{R}$ such that we have the following expressions for $\delta_{R}(i)$ and $\delta_{J}(i)$.

$$
\begin{gathered}
\delta_{R}(i)=\lambda f\left[\sum_{j=1}^{\min \left(W_{R}-1, i-1\right)} y_{i j}^{L}\left(W_{R}-j\right)+\sum_{j=1}^{\min \left(W_{R}-1, N-i\right)} y_{i j}^{R}\left(W_{R}-j\right)\right] \\
\xi(i, x)=\lambda f\left[\sum_{j=1}^{\min (x-1, i-1)} z_{i j}^{L} j+\sum_{j=1}^{\min (x-1, N-i)} z_{i j}^{R}\right]
\end{gathered}
$$

Here, the implication of this model is that the load changes are effectively captured by $W_{R}(i)$ and $W_{J}(i)$, and these parameters are (system dependent) relatively invariant with load changes. The uniform DQDB corresponds to the particular values $y_{i j}^{L}=y_{i j}^{R}=z_{i j}^{L}=z_{i j}^{R}=1$. In the present case, the impedance is given by $\Delta(i)=\xi\left(i, W_{J}(i)\right)+\delta_{R}(i)$.

In order that the system is fair, the parameters $y_{i j}^{L}, y_{i j}^{R}, z_{i j}^{L}$ and $z_{i j}^{R}$ (which are not independent of $i$ nor of each other) must satisfy the coupled system of equations $\{\Delta(i)=a\}_{i=1,2, \ldots, N}$ for every load $f$, where $a$ is a constant. Let $W_{J}(i)=W_{a}$ for $i=1,2, \ldots, N$ since the waiting times are the same. Now we have a simplified expression for $\Delta(i)=\xi\left(i, W_{a}\right)+\delta_{R}(i)$, whose analysis is simple since $\xi(i,$.$) is evaluated at fixed value W_{a}$ for the second operand. From Sections 4.1 and 4.2 , for $y_{i j}^{L}=y_{i j}^{R}=z_{i j}^{L}=z_{i j}^{R}=1$, recall that as the load is varied the profile of $\delta_{R}(i)$ is always a dome, but that of $\xi(i, x)$ is a dome at low loads and a bowl at high loads.

The parameters $y_{i j}^{L}, y_{i j}^{R}, z_{i j}^{L}$ and $z_{i j}^{R}$ can be imagined to be weights that multiply the terms of the profiles under unit parameters to yield $\Delta(i)$. Since at low loads, the profile under unit parameters must be a dome, to satisfy $\Delta(i)=a$ for $i=1,2, \ldots, N$, the effective distribution of the parameters must be a bow! (i.e. the parameters must provide more impedance for the nodes located at the ends compared to those at the middle). Since the network parameters are fixed, at higher loads these parameters will still provide same type of impedance to the end nodes; but such distribution will not be able to provide equal impedance since the distribution of $\delta_{J}(i)$ under unit parameters is a bowl. Thus the network will not be fair at loads that make $\delta_{J}(i)$ a bowl. This result can be shown by considering the parameters that provide equal impedance to all nodes at particular load and then considering the impedance as load changes. Consider a load $f_{1}$ such that $W_{R}<N / 2$ and $W_{J}(i)<N / 2$ for all $i$. Then consider the following assignment for the parameters.

\begin{tabular}{|l|c|c|}
\hline range for $i$ & $z_{i j}^{R}$ & $z_{i j}^{L}$ \\
\hline$i \in\left[1, W_{f_{1}}\right]$ & 1 & 1 \\
\hline$i \in\left[W_{f_{1}}, N / 2\right]$ & $\frac{W_{f_{1}}\left(W_{f_{1}}-1\right)}{i(i-1)}$ & 1 \\
\hline
\end{tabular}

\begin{tabular}{|l|c|c|}
\hline range for $i$ & $y_{i j}^{R}$ & $y_{i j}^{L}$ \\
\hline$i \in\left[1, W_{R}\right]$ & 1 & $\frac{W_{R}\left(W_{R}-1\right)}{2\left[W_{R}(i-1)-\frac{(i-1)}{2}\right]}$ \\
\hline$i \in\left[W_{R}, N / 2\right]$ & 1 & 1 \\
\hline
\end{tabular}

\footnotetext{
${ }^{5}$ The actual computation of these parameters from the physical description is not implied here.
} 
Using these parameters we obtain $\Delta(i)=\lambda f\left[W_{f_{1}}\left(W_{f_{1}}-1\right)+W_{R}\left(W_{R}-1\right)\right]$ for all $i=$ $1,2, \ldots, N$. When the load becomes $f_{2} \neq f_{1}$, such that $W_{f_{2}}=W_{f_{1}}+1$, simple calculation shows that $\Delta(i)$ is not the same for all $i=1,2, \ldots, N$.

If fairness is the only criterion, one can conceive of a method that adaptively delays the appropriate jobs based on the load. Implementation of such technique critically depends on our ability to accurately predict the load and the waiting times. The present work only provides estimates for the profiles, and more powerful system identification methods (Kushner and Clark [22], Benveniste et al. [4], Chen [7], Gerencser [17]) that can handle noise due to unmodelled dynamics are required to implement such adaptive algorithms.

\section{Conclusions}

We presented an approximate model to analyze the profiles of average waiting times at the nodes of a DQDB system. We illustrated an intimate relationship between the load and the fairness characteristics of the system by introducing the notion of working windows. The working window, whose value depends on the position of a node, expands or contracts in response to changes in the load. By considering a uniform model of the system, we study the waiting time profiles viewed as functions of the location of a node. Through a detailed analysis, we showed that there are only a finite number of distinct profiles for the waiting time. We showed that these profiles are variants of three basic profiles, and can be obtained by considering suitable ranges of nodes. Using a coarser analysis, we showed that there are only two dominant profiles for most of the load range, and all the other profiles occur only in a small range for the load. Furthermore, around a critical load, small changes in load cause the system to switch between these two basic modes. We also presented several simulation results that confirm our analytical results. This work can be viewed as an evidence for the inherent unfairness properties of systems with DQDB type of protocols, and in this respect provides motivation and justification for modifications that mke the system fair $[19,24,26]$.

We consider this work only as a first step in analyzing global behavior of DQDB as a function of load. Better approximations for this system are likely to reveal more richness in the variety of profiles for the waiting times. Also, DQDB can be studied for several other parameters such as throughput, effects of non-uniform job arrivals, etc.

\section{Appendix A: Profiles of $\delta_{R}(i)$}

The basic idea of estimating $\delta_{R}(i)$ involves summing up the request bits arriving from node $i+j$ within the time $W_{R}-j$ for $j=1,2, \ldots, \min \left(W_{R}-1, N-i\right)$. And we perform similar computation for the node $i-j, j=1,2, \ldots \min \left(W_{R}-1, i-1\right)$. We can imagine this process as two windows to the left and right of node $i$ (Fig. $3(\mathrm{a}))$. Here, $\min \left(W_{R}-1, i-1\right)$ and $\min \left(W_{R}-1, N-i\right)$ are called widths of left and right windows respectively. As explained in Section 3.2 , the general expression for $\delta_{R}(i)$ is given by

$$
\delta_{R}(i)=\lambda f\left[\sum_{j=1}^{\min \left(W_{R}-1, i-1\right)}\left(W_{R}-j\right)+\sum_{j=1}^{\min \left(W_{R}-1, N-i\right)}\left(W_{R}-j\right)\right]
$$


We consider the following cases:

(a) $W_{R}(i) \leq N / 2$ : Consider $i \in\left[W_{R}, N / 2\right]$ and in this case the windows spread for width of $W_{R}$ on either side. Thus we have

$$
\delta_{R}(i)=\lambda f\left[\sum_{j=1}^{W_{R}-1}\left(W_{R}-j\right)+\sum_{j=1}^{W_{R}-1}\left(W_{R}-j\right)\right]=\lambda f W_{R}\left(W_{R}-1\right)=W_{R}-1
$$

Consider $i \in\left[1, W_{R}\right]$ and in this case the right window spreads for width of $W_{R}$ and the left window spreads only for width of $i-1$. Thus we have

$$
\begin{aligned}
\delta_{R}(i) & =\lambda f\left[\sum_{j=1}^{i-1}\left(W_{R}-j\right)+\sum_{j=1}^{W_{R}-1}\left(W_{R}-j\right)\right]=\lambda f\left[2 \sum_{j=1}^{W_{R}-1}\left(W_{R}-j\right)-\sum_{j=1}^{W_{R}-i} j\right] \\
& =\lambda f\left[W_{R}\left(W_{R}-1\right)-\frac{\left(W_{R}-i\right)\left(W_{R}-i+1\right)}{2}\right] \\
& =W_{R}-1-\frac{f \lambda}{2}\left(W_{R}-i\right)\left(W_{R}-i+1\right)
\end{aligned}
$$

In summary, we have the following expression for $\delta_{R}(i)$.

$$
\delta_{R}(i)= \begin{cases}\left(W_{R}-1\right) & \text { if } i \in\left[W_{R}, N / 2\right] \\ W_{R}-1-\frac{\mu \lambda}{2}\left(W_{R}-i\right)\left(W_{R}-i+1\right) & \text { if } i \in\left[1, W_{R}\right]\end{cases}
$$

This function is monotonically increasing in the range $\left[1, W_{R}\right]$, constant in the range $\left[W_{R}, N-W_{R}\right]$ and monotonically decreasing in the range $\left[N-W_{R}, N\right]$.

(b) $W_{R}(i) \geq N$ : In this case, the left window has a width of $i-1$ and the right window has a width of $N-i$. Thus we have

$$
\delta_{R}(i)=\lambda f\left[\sum_{j=1}^{i-1}\left(W_{R}-j\right)+\sum_{j=1}^{N-i}\left(W_{R}-j\right)\right] .
$$

After straight forward algebraic simplification we obtain the following equation.

$$
\delta_{R}(i)=(N-1)-\frac{f \lambda}{2}\left[N(N+1)-2(N+1) i+2 i^{2}\right]
$$

This function is monotonically increasing in the range $[1,(N-1) / 2]$ and is monotonically decreasing in the range $[(N+2) / 2, N]$.

(c) $N / 2<W_{R}(i)<N$ : Consider $i \in\left[1, N-W_{R}\right]$, its left window has a width of $i-1$ and right window has a width of $W_{R}$. This case is similar to (a). Now consider $i \in\left[N-W_{R}, W_{R}\right]$. This node has $W_{R}$ to be the widths of both left and right windows. Thus in this range it is similar to the case (b). Thus we have the following expression for $\delta_{R}(i)$.

$$
\delta_{R}(i)= \begin{cases}\left(W_{R}-1\right)-\frac{f \lambda}{2}\left(W_{R}-i\right)\left(W_{R}-i+1\right) & \text { if } i \in\left[1, N-W_{R}\right] \\ (N-1)-\frac{\lambda f}{2}\left[N(N+1)-2(N+1) i+2 i^{2}\right] & \text { if } i \in\left[N-W_{R}, N / 2\right]\end{cases}
$$

Thus this function is monotonically increasing until the middle and then monotonically decreasing until the end.

In summary, the overall profile of $\delta_{R}(i)$ is always convex or a dome. 


\section{Appendix B: Profiles of $\xi(i, x)$}

Computation of $\xi(i, x)$ is similar to that of $\delta_{R}(i)$ because we have to consider a window of fixed size $x$. The main difference is that the profile of the windows is as shown in Fig. 3(b). Thus we have

$$
\xi(i, x)=\lambda f\left[\sum_{j=1}^{\min (x-1, i-1)} j+\sum_{j=1}^{\min (x-1, N-i)} j\right] .
$$

Notice that the nodes located away from $i$ contribute more to the impedance at node $i$ than those located near; this aspect is in contrast to $\delta_{R}(i)$ where the nodes near to $i$ contribute more to the impedance than those farther away.

(a) $x \leq N / 2$ : Consider $i \in[x, N / 2]$ and in this case the windows spread for width of $x$ on either side. Thus we have

$$
\xi(i, x)=\lambda f\left[\sum_{j=1}^{x-1} j+\sum_{j=1}^{x-1} j\right]=\lambda f x(x-1)
$$

Consider $i \in[1, x]$ and in this case the right window spreads for width of $x$ and the left window spreads only for width of $i-1$. Thus we have

$$
\xi(i, x)=\lambda f\left[\sum_{j=1}^{i-1} j+\sum_{j=1}^{x-1} j\right]=\lambda f\left[\frac{i(i-1)}{2}+\frac{x(x-1)}{2}\right]
$$

In summary, we have the following expression for $\xi(i, x)$.

$$
\xi(i, x)= \begin{cases}f \lambda x(x-1) & \text { if } i \in[x, N / 2] \\ f \lambda\left[\frac{i(i-1)}{2}+\frac{x(x-1)}{2}\right] & \text { if } i \in[1, x]\end{cases}
$$

(b) $x \geq N$ : In this case both the left window has a width of $i-1$ and the right window has a width of $N-i$. Thus we have

$$
\xi(i, x)=\lambda f\left[\sum_{j=1}^{i-1} j+\sum_{j=1}^{N-i} j\right]
$$

After straight-forward algebraic simplification we obtain the following equation.

$$
\xi(i, x)=\frac{f \lambda}{2}\left[2 i^{2}-2 i(N+1)+N(N+1)\right]
$$

This function is monotonically decreasing in the range $[1,(N-1) / 2]$ and is monotonically increasing in the range $[(N+2) / 2, N]$. Contrast this behavior with the case (b) of $\delta_{R}(j)$ which has a profile of opposite nature.

(c) $N / 2 \leq x \leq N$ : Consider $i \in[1, N-x]$, its left window has a width of $i-1$ and right window has a width of $x$. This case is similar to (a). Now consider $i \in[N-x, x]$. This node has $x$ to be the widths of both left and right windows. Thus in this range it is similar to the case (b).

$$
\xi(i, x)= \begin{cases}f \lambda\left[\frac{i(i-1)}{2}+\frac{x(x-1)}{2}\right] & \text { if } i \in[1, N-x] \\ \frac{f \lambda}{2}\left[2 i^{2}-2 i(N+1)+N(N+1)\right] & \text { if } i \in[N-x, N / 2]\end{cases}
$$




\section{Appendix C}

We establish two approximations that are used in deriving bounds for $W_{J}(i)$. We shall prove that

$$
(1+x)^{\frac{1}{2}} \leq 1+1 / 2 x
$$

for all values of $x \geq-1$. And for $x \leq K$

$$
(1+x)^{\frac{1}{2}} \geq 1+\frac{x}{1+\sqrt{1+K}}
$$

To prove equation (C.1), first note that $\left(1+\frac{1}{2} x\right)^{2}=1+x+x^{2} / 4 \geq 1+x$, and then take square root on both sides.

Consider equation C.2. By squaring both sides and adjusting the terms, we can show that this condition is equivalent to $(\sqrt{K+1}+1)(\sqrt{K+1}-1) \geq x$, which in turn is equivalent to the hypothesis $x \leq K$.

\section{Appendix D: Two Systems of Equations}

We consider types of equations of tue following kind

$$
\begin{gathered}
{[W(i)-1]^{2}=X(i)+A W(i)[W(i)+1]} \\
{[W(i)-1]^{2}=X(i)+A}
\end{gathered}
$$

where $W(i)$ and $X(i)$ are functions of $i$ and $A$ does not depend on $i$. Then bounds of $W(i)$ can be expressed as follows:

$$
\begin{aligned}
& W(i) \leq B X(i)+C \\
& W(i) \geq D X(i)+E
\end{aligned}
$$

where $B, C, D$ and $E$ do not depend on $i$. Thus, we have $W(i)=\Theta(X(i))$.

First consider the equation (D.2) which can be expressed by a quadratic equation as $(W(i))^{2}-2 W(i)+(1-X(i))$ whose solution can be written as $W(i)=1 \pm \sqrt{1-(1-X(i))}$. By using the result of Appendix $\mathrm{C}$, we can write $W(i) \leq 1+(1-1 / 2(1-X(i)))=X(i) / 2+3 / 2$. Since $\Delta(i)$ is bounded, we have $X(i)$ bounded; thus, $[1-(1-X(i))]^{1 / 2} \geq 1-\frac{(1-X(i)}{a}$ $=1 / a X(i)+(1-1 / a)$ for some constant $a$. Now we can conclude that $W(i) \geq D X(i)+E$.

Now consider the equation (D.1), which can be expressed by a quadratic equation (1 $A)[W(i)]^{2}-[2+A] W(i)+[1-X(i)]=0$ for $A<1$. The solution of this equation can be written as

$$
W(i)=\frac{A+2}{2(1-A)} \pm 1 / 2 \sqrt{1+\frac{6 A+3-4(1-A)(1-X(i))}{(1-A)^{2}}}
$$

Now by using the result of Appendix $C$, we obtain

$$
W(i) \leq \frac{A+2}{2(1-A)}+1 / 2\left[1+\frac{6 A+3+4(1-A)(X(i)-1)}{(1-A)^{2}}\right]=B X(i)+C
$$

Similar result can be shown for the case $A>1$. By using the boundedness of $W(i)$, we can show that $W(i) \geq D X(i)+E$. In summary, given equations of type $D .1$ or $D .2$, we can express the upper and lower bounds of $W(i)$ as linear functions of $X(i)$. 


\section{Appendix E: Coarse Analysis for $W_{J}(i)$}

Because of the monotonic relation between $W_{J}(i)$ and $\Delta(i)$, we subsequently consider only. $\Delta(i)$ for ease of presentation. Based on the range of values for $\bar{W}$ we have three distinct profiles for $\Delta(i)$ plotted as a function of the node number $i$; here we express $\Delta(i)=\delta^{+}(i)+$ $\delta^{-}(i)$, where $\delta^{+}(i)$ and $\delta^{-}(i)$ are the impedance factors (including the parts due to jobs and request bits) due to the two buses. The profile of $\Delta(i)$ is as follows.

(a) $\bar{W} \leq N / 2$ : For the nodes in the interval $[1, \bar{W}]$ the profile is increasing, in the range $[\bar{W}, N-\bar{W}]$ it is constant, and in the range $[N-\bar{W}, N]$ it is decreasing.

(b) $N / 2<\bar{W}<N$ : In the interval $[1, N-\bar{W}]$ the profile is increasing, and in the interval $[\bar{W}, N]$ it is decreasing. In the middle portion, the plot is concave (as in Case (c)).

(c) $\bar{W} \geq N$ : The plot consists of a single concave curve.

Here we use an estimation method different from that of Appendices A and B, although both are still based on the working window characterization. This method is coarse in that we use same window size for all node and also use the same size for the request and job windows. It is also more detailed in that we take into account the probability with which a job arriving at one node is sent in the direction of another node. It is interesting to note that this variation also essentially confirms the same basic overall beahavior of the profile.

\section{E.1. Low to Medium Loads}

We consider the loads such that $\bar{W}<N / 2$ which is equivalent to $f<1-2 / N$. Consider a station $i \in[W, N-\bar{W}]$. The opposition factor due to one bus is given by the following equation.

$$
\begin{aligned}
\delta^{+}(i) & =\delta_{J}^{+}(i)+\delta_{R}^{+}(i) \\
& =\sum_{j=1}^{\bar{W}-1} \frac{j(N-i+j) \lambda f}{N-1}+\sum_{j=1}^{\bar{W}-1} \frac{j(N-i-j) \lambda f}{N-1} \\
& =\frac{2 \lambda f}{N-1} \sum_{j=1}^{\bar{W}}(N-i) j=\frac{\lambda f(N-i) \bar{W}(\bar{W}-1)}{N-1}
\end{aligned}
$$

Here the first term $\delta_{J}^{+}(i)$ is due to the oncoming jobs and the second term $\delta_{R}^{+}(i)$ is due to the request bits. Compared to the expressions in Appendices $\mathrm{A}$ and $\mathrm{B}$, here there is an averaging term $\frac{N-i+j}{N-1}$ for the effective arrival rate seen by node $i$ due to node $j$. Consider bus with slots flowing from node 1 to node $N$. At node $i$, the jobs generated at a node $i-j, j>0$, that are destined to the nodes in the interval $[i-j+1, N]$ will contribute to $\delta_{J}^{+}(i)$; a job at node $i-j$ has a probability of $\frac{N-i+j}{N-1}$ of being sent on the present bus. Hence, its effective arrival rate at node $i-j$ is multiplied this factor in the first term of $\delta_{J}^{+}(i)$. For the same bus, the jobs arriving at node $i+j, j>0$, that are destined to the nodes in the interval $[i+j+1, N]$ contribute to $\delta_{R}^{+}(i)$; note here that the jobs destined to nodes in the interval $[1, i+j-1]$ do not contribute to $\delta_{R}^{+}(i)$. 
Similarly opposition factor due to the traffic in the other direction is given by

$$
\delta^{-}(i)=\frac{\lambda f(i-1) \bar{W}(\bar{W}-1)}{N-1}
$$

Considering both the opposition factors, the total opposition factor is given by

$$
\Delta(i)=\delta^{+}(i)+\delta^{-}(i)=\lambda f \bar{W}(\bar{W}-1) .
$$

Consider the case $1 \leq i \leq \bar{W}$, and the case $N-\bar{W} \leq i \leq N$ is similar. We have

$$
\begin{aligned}
& \delta^{+}(i)= \sum_{j=1}^{i-1} \frac{j(N-i+j) \lambda f}{N-1}+\sum_{i=1}^{W-1} \frac{j(N-i-j) \lambda f}{N-1} \\
& \delta^{-}(i)= \sum_{j=1}^{W-1} \frac{j(i+j-1) \lambda f}{N-1}+\sum_{i=1}^{i-1} \frac{j(i-j-1) \lambda f}{N-1} \\
& \Delta(i)=\lambda f\left[\frac{i(i-1)}{2}+\frac{\bar{W}(\bar{W}-1)}{2}\right]
\end{aligned}
$$

In the region $[1, \bar{W}]$ the profile of $\Delta(i)$ is a parabola around the axis of $i$. This predicted shape, which is convex, matches our simulation results in Fig. 15(a).

\section{E.2. Overload}

We consider the loads where $\bar{W} \geq N$, i.e., $f \geq 1-2 / N$. Here the window expands all the way across the stations. Thus we have

$$
\begin{aligned}
\delta^{+}(i) & =\sum_{j=1}^{i-1} \frac{j(j-1) f \lambda}{n-1}+\sum_{j=i+1}^{N} \frac{(N-j)(j-i) f \lambda}{N-1} \\
& =\frac{2 i(i-1)(i-2) f \lambda}{6(N-1)}+\frac{f \lambda}{6(N-1)}\left[N^{3}-N+(1-3 N) i+3 i^{2}(1-N)+2 i^{3}\right] \\
& =\frac{f \lambda}{6(N-1)}\left[2 i^{3}-3 i^{2}(1-N)-i\left(3 N^{2}+6 N-7\right)+2\left(N^{3}-N\right)\right] .
\end{aligned}
$$

This quantity attains its minimum at $i$ corresponding to $\frac{\partial \delta^{+}(i)}{\partial i}=0$ which yields $6 i^{2}+$ $6 i(N-1)-\left(3 N^{2}+6 N-7\right)=0$. The solution to this quadratic equation is given by

$$
i_{\min }=\frac{-(N-1)}{2}+\sqrt{\frac{(N-1)^{2}}{4}+\frac{3 N^{2}+6 N-7}{6}}=\frac{3 N+1}{\sqrt{6}} .
$$

We can also consider a better approximation by considering the quantity under the square root sign as follows

$$
\frac{N^{2}-2 N+1}{4}+\frac{3 N^{2}+6 N-7}{6} \leq \frac{3\left(N^{2}+N\right)}{4} \leq 3 / 4(N+1)^{2} .
$$


Using this we obtain $i_{\min } \leq 0.35 N$. Note that the minima does not occur at the middle. This indicates that the upcoming jobs will have more effect on the station than the jobs down stream. Now consider $\Delta(i)$.

$$
\begin{aligned}
\Delta_{(i)}(i) & \delta^{+}(i)+\delta^{-}(i) \\
= & \frac{f \lambda}{N-1}\left[\sum_{j=1}^{i-1} j(N-i+j)+\sum_{j=1}^{N-i} j(N-i-j)\right] \\
& +\frac{f \lambda}{N-1}\left[\sum_{j=1}^{i-1} j(i-j-1)+\sum_{j=1}^{N-i} j(i+j-1)\right] \\
= & f \lambda\left[\sum_{j=1}^{i-1} j+\sum_{j=1}^{N-i} j\right]=\frac{f \lambda}{2}\left[2 i^{2}-2(N+1) i+N(N+1)\right]
\end{aligned}
$$

It is interesting to note that $\Delta(i)$ is a second degree polynomial in $i$ even though $\delta^{+}(i)$ and $\delta^{-}(i)$ are third degree polynomials in $i$. It is easy to check that $\Delta(i)$ attains a minima at $i=\frac{N+1}{2}$ and $\Delta(1)=\Delta(N)=\frac{\lambda f}{2}[N(N-1)]$. This case, which yields concave profiles, is confirmed by our simulation results in Fig. 15.

\section{E.3. Medium to High Loads}

We consider the intervals $[1, N-\bar{W}],[N-\bar{W}, \bar{W}]$ and $[\bar{W}, N]$. We have the following equations for this case.

$$
\Delta(i)= \begin{cases}\frac{\lambda f}{2}[i(i-1)+\bar{W}(\bar{W}-1)] & \text { if } i \in[1, N-\bar{W}] \\ \frac{\lambda f}{2}\left[2 i^{2}-2(N+1) i+N(N+1)\right] & \text { if } i \in[N-\bar{W}, \bar{W}]\end{cases}
$$

Notice that in this Appendix E, we only have three basic profiles as opposed to at least twenty four in Section 4.3; this is because of the coarser approximation employed here. Also the analysis here is much simpler because we employ $\bar{W}$ as the window size.

\section{References}

[1] American National Standard, Fiber Distributed Data Interface (FDDI) token ring media access control, ANSI X3.139-1987, November 5, 1987.

[2] IEEE Standard 802.6-1990, IEEE Standards for Local and Metropolitan Area Networks: Distributed Queue Dual Bus (DQDB) Subnetwork of a Metropolitan Area Network (MAN), New York:IEEE, July 3, 1991.

[3] M. Abramowitz and I.A. Stegun (Eds.), Handbook of Mathematical Functions, Dover Pub., New York, 1965.

[4] A. Benveniste, M. Metivier, P. Priouret, Adaptive Algorithms and Stochastic Approximation, Springer-Verlag, New York, 1990. 
[5] C. Bisdikian, Waiting time analysis in a single buffer DQDB (802.6) network, IEEE J. Selected Areas in Communication, vol. 8, no.8, 1990, pp. 1565-1573.

(6) C. Bisdikian, A queueing model with applications to bridges and the DQDB (IEEE 802.6) MAN, Computer Networks and ISDN Systems, vol. 25, 1993, pp. 1279-1289.

[7] H.-F. Chen, Recusrsive Estimation and Control of Stochastic Systems, John Wiley, New York, 1985.

[8] M. Conti, E. Gregori and L. Lenzini, DQDB media access control protocol: Performance evaluation and unfairness analysis, Proc. 3rd IEEE Workshop on Metropolitan Area Networks, San Diego, CA, mar 28-30, 1989, pp. 7.3.1-7.3.33.

[9] P. Davids and P. Martini, Performance analysis of DQDB, 9th Annual International Phoenix Conference on Computers and Communications, March 21-23, 1990, Scottsdale, Arizona, pp. 548-555.

[10] P. Davids and T. Welzel, Performance analysis of DQDB based on simulation, 3rd IEEE Workshop on Metropolitan Area Networks, March 1989.

[11] P. J. Denning, The working set model for program behavior, Communications of ACM, vol. 11, no. 5, 1968, pp. 323-333.

[12] P. J. Denning, Working sets past and present, IEEE Trans. on Software Engineering, vol. SE-6, no.1, 1980, pp. 64-84.

[13] S. Fdida and H. Santoso, Performance issues of DQDB, 4th International Conference on Data Communication Systems and Their Performance, Barcelona, June 20-22, 1990.

[14] S. Fdida and H. Santoso, Approximate performance model and fairness condition of the DQDB protocol, NATO Advanced Research Workshop on Architecture and Performance Issues of High-Capacity Lans and Mans, Sophia-Antipolis, June 1990 (to be published by Springer-Verlag),

[15] J. Filipiak, Access protection for fairness in a DQDB MAN, Proc. IEEE Int. Conf. Communications, Boston, MA, 1989, pp. 635-639.

[16] M.W. Garrett, and S. Li, A Study of slot reuse in dual bus multiple access networks, Proc. of IEEE INFOCOM'90, 1990, pp. 617-629.

[17] L. Gerencser, Strong approximation results in estimation and adaptive control, in Topics in Stochastic Systems: Modelling, Estimation and Adaplive Control, Springer-Verlag, 1992.

[18] P. T-. Gia and T. Stock, Approximate performance analysis of the DQDB access protocol, Computer Networks and ISDN Systems, vol. 20, 1990, pp. 231-240.

[19] E. L. Hahne, A.K. Choudhury and N.F. Maxemchuk, Improving the fairness of Distributed-Queer-Dual-Bus Networks, Proc. of IEEE INFOCOM'90, 1990, pp. 175-184. 
[20] E. Y. Huang and L. F. Merakos, On the access fairness of the DQDB MAN protocol, 9th Annual International Phoenix Conference on Computers and Communications, March 21-23, 1990, Scottsdale, Arizona, pp. 556-559.

[21] H. Kaur and G. Campbell, DQDB - an access delay analysis, Proc. of IEEE INFO. COM'90, 1990, pp. 630-635.

[22] H. J. Kushner and D. S. Clark, Stochastic Approximation Methods for Constrained and Unconstrained Systems, Springer Verlag, New York, 1979.

[23] A. Lombardo, S. Palazzo, D. Panno, R. Pingnatelli, and L. Susanna, An adpative policing mechanism for a DQDB MAN, Computer Networks and ISDN Systems, vol. 25, 1993, pp. 1119-1126.

[24] Y. S. Leu and D. H. C. Du, Cycle compensation DQDB: A completely fair multiple priority class protocol for the uni-directional twin-bus architecture, Tech. Rep. TR 90-32, Computer Science Dept., University of Minnesota, May 1990.

[25] J. F. Mollenauer, Standards for metropolitan area networks, IEEE Communications Magazine, vol. 26, 1988, pp. 15-19.

[26] B. Mukherjee and S. Banerjee, Alternative strategies for improving the fairness in and an analytical model of the DQDB network, IEEE Trans. on Computers, vol. 42, no. 2, 1993, pp. 151-167.

[27] B. Mukherjee and C. Bisdikian, A journey through the DQDB network literature, Performance Evaluation, vol. 16, no. 1-3, 1992, pp. 129-158.

[28] A. Myles, DQDB simulation and MAC protocol analysis, Electronic Letters, April 27, 1989, vol, 25, mo. 9, pp. 616-618.

[29] R.M. Newman and J.L. Hullet, Distributed queueing: A fast and efficient packet access protocol for QPSX, in New Communication Services: A Challenge to Computer Technology, P. Kuhn (Ed.), North-Holland 1986, pp. 294-299.

[30] R.M. Newman, Z.L. Budrikis and J.L. Hullet, The QPSX man, IEEE Communications Magazine, vol. 26, no. 4, 1988, pp. 20-28.

[31] S. N. Rasband, Chaotic Dynamics of Nonlinear Systems, John Wiley, New York, 1990.

[32] M. A. Roddrigues, Erasure node: Performance improvements for the IEEE 802.6 MAN, Proc. of IEEE INFOCOM'90, 1990, pp. 636-643.

[33] K. Sauer, and W. Schodl, Performance aspects of the DQDB protocol, Computer Networks and ISDN Systems, vol. 20, 1990, pp. 253-260.

[34] J. W. Wong, Throughput of DQDB networks under heavy load, Proc. 7th Europ. Fibre Optic Commun. Local Area Networks Expo, Amsterdam, The Netherlands, June 1989, pp. 146-151. 
[35] T. Yokotani, H. Sato, S. and Nakatsuka, A study on a performance improvement algorithm in DQDB MAN, Computer Networks and ISDN Systems, vol. 25, 1993, pp. 11071117.

[36] M. Zuckerman, On packet switching capacity in QPSX, Proc. of IEEE/IEICE Global Telecommunications Conf., 1987, pp. 15-18.

[3i] M. Zuckerman, Queueing performance of QPSX, Proc. 12th Int. Teletraffic Congress, 1988, pp. 2.2B6.1-2.2B6.7.

[3S] M. Zuckerman, Overload control of isochronous traffic in QPSX, Proc. IEEE Global T'elecommunications Conf., 1988, pp. 38.3.1-38.3.5.

[39] M. Zuckerman, and P. G. Potter, The DQDB protocol and its performance under overload traffic conditions, Computer Networks and ISDN Systems, vol. 20, 1990, pp. 261-270. 


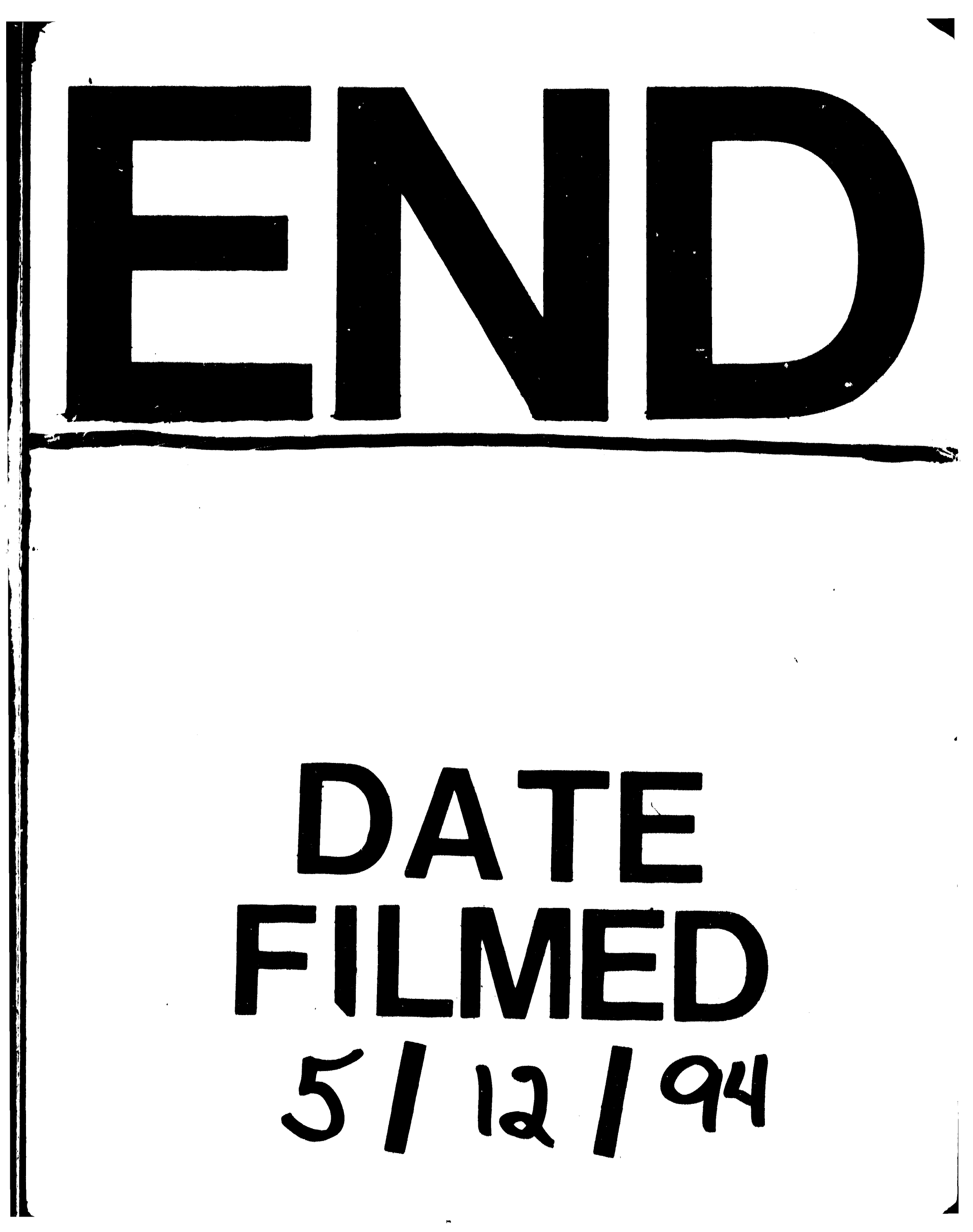




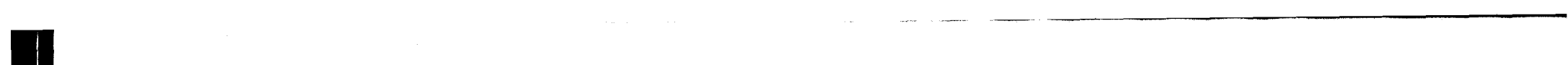

